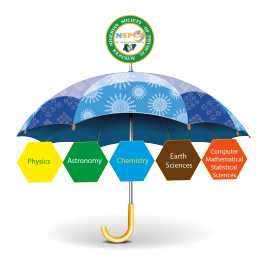

\title{
A Modified Forced Randomized Response Model
}

\author{
A. T. Adeniran*, A. A. Sodipo, C. G. Udomboso \\ Department of Statistics, University of Ibadan, Ibadan, Nigeria.
}

\begin{abstract}
This paper proposed a new Randomized Response Model (RRM) to estimate proportion of people characterizing a sensitive variable ( $S$ ) under study. Simple random sampling with replacement and stratified simple random sampling scheme were adopted. Maximum likelihood and Bayesian estimation procedures of the proposed model were developed and compared. The sampling distribution (expectation and variance) of the proposed estimator under the two sampling techniques, efficiency comparison of the proposed model with some existing models, and numerical illustration of all the compared models were also explored. The study found that the proposed model outperformed other existing RRMs in terms of efficiency and it proved to be more protective in designing survey for sensitive related issues.
\end{abstract}

Keywords: Randomized response, proportion, sensitive variable, maximum likelihood estimator, sampling distribution.

Article History :

Received: 25 December 2019

Received in revised form: 15 February 2020

Accepted for publication: 17 February 2020

Published: 28 February 2020

(c)2020 Journal of the Nigerian Society of Physical Sciences. All Rights Reserved. Communicated by: T. Latunde

\section{Introduction}

Surveys usually collect responses to a large number of items from each sample unit and many institutions use empirical evidence from surveys to make their policies. Thus, surveys play a prominent role in society, hence collecting and interpreting survey data correctly is essential. One of the most obvious problems in census or sample surveys is the inability of the researcher to collect responses on some or all of the items for a sampled unit or when some responses are deleted because they fail to satisfy edit constraints [1]. In the vernacular of sample survey, this is the problem of non-response. Non response can arise for a variety of reasons, one of which is the sensitive nature of the survey question.

Socially sensitive questions such as shoplifting, rash driving, tax evasion, felony, false declaration of assets, expenditure on addiction of various form (drug abuse), kidnapping, occult affiliation, psychosocial status, cheating in national examination;

${ }^{*}$ Corresponding Author Tel. No: +2348035529045

Email address: at.adeniran@mail .ui.edu.ng (A. T. Adeniran) or health habits and sexual orientation related questions such as HIV infection, induced abortion, masturbation, ravish, homosexuality, and other illegal, unethical, prohibited attitudes or practices that receive disapproval by the society are thought to be threatening to respondents [2-3]. When sensitive topics are studied, respondents often react in ways that negatively affect the validity of data by giving socially desirable answers to avoid social embarrassment and to project a positive self-image [4-5]. The Randomized Response Model (RRM) also known as Randomized Response Technique (RRT) or Randomized Response Distribution/Design (RRD) is a survey method specifically developed to improve the participation and precision of answers to sensitive questions because the frequency of socially undesirable, disgraceful, embarrassing, incriminating, ignominious, highly stigmatizing variable has been found usually underestimated in surveys.

In the year 1965, Warner explained that the reluctance of the respondents to elicit sensitive or probably harmful information would diminish when respondents are convinced that their anonymity be guaranteed, when incriminating answers could be covered 
even from the interviewer, the need to present oneself in a positive way would decrease and honest answering would increase [6]. Following this assumption, [7] did the pioneering work on a RRT which required the interviewee to give a "yes"or "no"answer either to the sensitive question or to its negative depending on the outcome of a randomizing device not revealed to anybody including the interviewer. After [7] original RRM, different authors developed various RR models including Unrelated Questioning Technique (UQT), Forced Randomized Response Model (FRRM), stratification of new and existing models in Randomized Response (RR) surveys, calibration techniques and so on. According to [8-9] "FRRM and UQT with known population prevalence of the innocuous attribute are the best, a major setback is that it is not easy to come up with unrelated question with known prevalence and variance near zero, hence, UQT seems harder to be adopted." To circumvent this problem, this study proposed a Modified Forced Randomized Response Model (MFRRM) with a definite no response to the unrelated question.

The structure of the paper is as follows: In section 2, literatures that are related to the study are reviewed to identify all existing works, their contributions and limitations. Section 3 introduces the newly proposed RRM namely modified forced randomized response model. In the same section, Maximum Likelihood (ML) and Bayesian Estimator (BE) of the proposed RRM are explored, comprehensive treatment of statistical properties of the proposed estimator such as: unbiasedness, efficiency comparison with existing models, and cost efficiency of the proposed design are as well carried out. Section 4 presents analysis of the simulated data sets, discussion of results, and the concluding remarks. Tables and charts showing the results are also presented in this section.

\section{Existing Models}

This section lucidly renders a vivid review of related previous studies, starting with Warner original model, via intermediate models to the current advanced stage of robust quantitative RRM.

\subsection{Warner Randomized Response Model}

In Warner's (1965) original design, respondents are provided with a randomizer (say, spinner) and instructed to answer one of two statements:

(i) I belong to the sensitive group (selected with probability $p$ ).

(ii) I do not belong to the sensitive group (selected with probability $1-p$ ).

Respondents then in turn answer "true"or "not true"or "yes"or "no"according to their status on sensitive question without revealing to the interviewer which statement was selected by the randomizer. The probability tree diagram below illustrates the randomization procedure of [7] original randomized response model.

From Figure 1 above, the probability of yes and no answers are $\lambda=p \pi_{s}+(1-p)\left(1-\pi_{s}\right)$ and $1-\lambda=p\left(1-\pi_{s}\right)+(1-p) \pi_{s}$, respectively. Suppose $n_{o}$ and $n-n_{o}$ denote the total number of "yes" and "no"answers in the sample of $n$ respondents, following the maximum likelihood principle, the likelihood function to maximize is

$$
P(\lambda, n)=\left(\begin{array}{c}
n \\
n_{o}
\end{array}\right)\left[p \pi_{s}+(1-p)\left(1-\pi_{s}\right)\right]^{n_{o}}\left[p\left(1-\pi_{s}\right)+(1-p) \pi_{s}\right]^{n-n_{o}} .(1)
$$

Setting the derivative (w.r.t. $\pi_{s}$ ) of the natural logarithm of equation (1) to zero, Warner unbiased estimator of $\pi_{s}$ is

$$
\hat{\pi}_{s}=\frac{\frac{n_{o}}{n}-(1-p)}{2 p-1}=\frac{\hat{\lambda}-(1-p)}{2 p-1}, \quad p \neq \frac{1}{2}
$$

with variance

$$
\operatorname{Var}\left(\hat{\pi}_{s}\right)=\frac{\pi_{s}\left(1-\pi_{s}\right)}{n}+\frac{p(1-p)}{n(2 p-1)^{2}}
$$

where,

$n=$ number of observed respondents' (sample size),

$p=$ probability of selecting (and answering) sensitive question, and

$\pi_{s}=$ proportion of yes answers to the sensitive question.

Subsequently, both in theory and application, several other authors have suggested various alternatives RR models including [10-17] among many others. Recently, [18] used [7] RRM to measure corruption among public bureaucrats in Bolivia, Brazil, and Chile.

\subsection{Forced Randomized Response Model (FRRM)}

The forced response method otherwise referred to as UQT with known $\pi_{u}$ was originated by [12] and later simplified by [19] with the assumption that $\pi_{u}$ is a predetermined parameter. That is, $\pi_{u}$ exists or must be known either from independent ad-hoc studies or statistical abstracts. This assumption does not always hold. Even though $\pi_{u}$ is available in any register or statistical abstracts, cost of retrieving from records may be expensive. If $\pi_{u}$ is known beforehand, solving for $\pi_{s}$ in [11] model, and replacing $\lambda$ with its sample estimator $\left(\hat{\lambda}=\frac{n_{o}}{n}\right)$ gives

$$
\hat{\pi}_{s}=\frac{1}{p}\left[\hat{\lambda}-(1-p) \pi_{u}\right]=\frac{1}{p}\left[\frac{n_{o}}{n}-(1-p) \pi_{u}\right]
$$

with variance

$$
\begin{aligned}
& \operatorname{Var}\left(\hat{\pi}_{s}\right)=\frac{\pi_{s}\left(1-\pi_{s}\right)}{n}+\frac{(1-p)}{n p}\left[\pi_{s}\left(1-\pi_{u}\right)+\pi_{u}\left(1-\pi_{s}\right)\right. \\
& \left.+\frac{(1-p) \pi_{u}\left(1-\pi_{u}\right)}{p}\right] .
\end{aligned}
$$

Estimating $\pi_{s}$ in this manner is termed "Forced Randomized Response Model or Technique (FRRM or FRRT)". Among applied researchers, FRRM is the most famous design. Illegal 


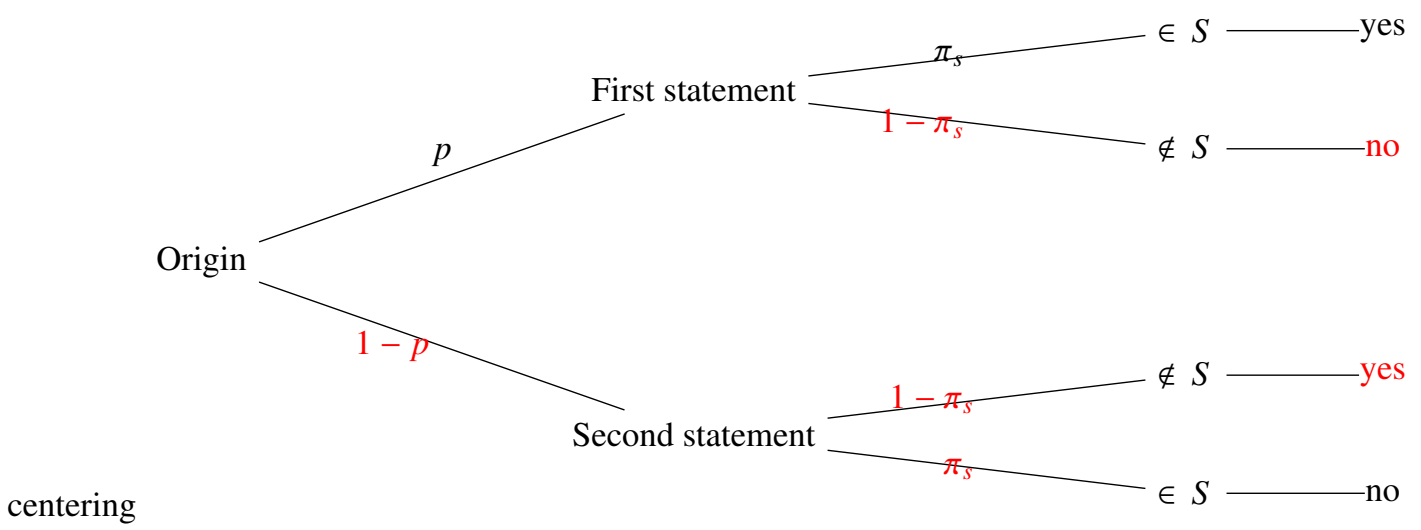

Figure 1: Probability tree diagram of the Warner original RRM

waste disposal [9], prevalence of civilian cooperation with militant groups in southeastern Nigeria [20], vote choice regarding a Mississippi abortion referendum [21], use of performance enhancing drugs [22], xenophobia and anti-Semitism in Germany [23], illegal poaching among South African farmers [24], and violation of regulatory laws by commercial firms [25] are few evidences among numerous practical application of the ForcedRR-UQT.

\subsection{Mangat Improved Two Step Procedure}

Alternative form of forced response model was developed by [15] as an optimization of one of his earlier designs. Mangat's procedure requires all respondents that have sensitive attribute $(S)$ to answer truthfully without use of randomizer. All respondents who do not have the sensitive attribute are required to use the randomizer to choose which from Warner's statements. This means that all no-answers are true negatives, and that only the yes answers are contaminated. [15] estimator of $\pi_{s}$ is

$$
\hat{\pi}_{s}=\frac{1}{p}\left(\frac{n_{o}}{n}-1+p\right)=\frac{1}{p}(\hat{\lambda}-1+p)
$$

with variance

$$
\operatorname{Var}\left(\hat{\pi}_{s}\right)=\frac{\pi_{s}\left(1-\pi_{s}\right)}{n}+\frac{(1-p)\left(1-\pi_{s}\right)}{n p} .
$$

Even though Mangat's estimator is most efficient when compared with the earlier similar estimators or models, his design is unrealistic as respondents with the sensitive attribute will be more inclined to lie, making the population estimates less valid, and this causes the results and inferences of his procedure to be less trustworthy [8], [26]. Recently, [27] modified and applied [15] RRM to estimate two sensitive attributes simultaneously.

\subsection{Stratified Warner's Randomized Response Model}

[16] presented a stratified RRT of [7] original model using an optimal allocation which is more efficient and cost effective than using a proportional allocation of [2] technique. The maximum likelihood estimate of $\pi_{s}$ using [16] RRM is

$$
\begin{aligned}
& \hat{\pi}_{s}=\sum_{h=1}^{L} W_{h} \hat{\pi}_{s h}=\sum_{h=1}^{L} W_{h}\left[\frac{\frac{n_{o h}}{n_{h}}-\left(1-p_{h}\right)}{2 p_{h}-1}\right] \\
& =\sum_{h=1}^{L} W_{h}\left[\frac{\hat{\lambda}_{h}-\left(1-p_{h}\right)}{2 p_{h}-1}\right]
\end{aligned}
$$

and the minimal variance of $\hat{\pi}_{s}$ is given by

$$
\operatorname{Var}\left(\hat{\pi}_{s}\right)=\frac{1}{n}\left[\sum_{h=1}^{L} W_{h}\left\{\pi_{s h}\left(1-\pi_{s h}\right)+\frac{p_{h}\left(1-p_{h}\right)}{\left(2 p_{h}-1\right)^{2}}\right\}^{\frac{1}{2}}\right]^{2}
$$

where, $W_{h}=\frac{N_{h}}{N}=$ stratum weight, $\hat{\lambda}_{h}=\frac{n_{o h}}{n_{h}}=$ proportion of yes-answer in a stratum $h, p_{h}=$ probability that a respondent in the sample stratum $h$ has a sensitive question (S) card, and $\hat{\pi}_{s h}=\frac{\hat{\lambda}_{h}-\left(1-p_{h}\right)}{2 p_{h}-1}=$ proportion of respondents with the sensitive trait in a stratum $h$, for $h=1,2, \cdots, L$.

\subsection{Mixed Stratified Randomized Response Model}

[17] proposed mixed stratified RRM by taking two independent samples of sizes $n_{1}$ and $n_{2}$. Also, two randomization devices $R_{1}$ and $R_{2}$ were used in each sample to estimate Human Immune Virus (HIV) seroprevalence rate in Kaduna State, Nigeria. Following the same maximum likelihood estimation procedure, an unbiased mixed stratified seroprevalence rates estimator is given by

$$
\hat{\pi}_{s}=\sum_{h=1}^{L} W_{h} \hat{\pi}_{s h}=\sum_{h=1}^{L} W_{h}\left[\frac{n_{h 1}}{n_{h}} \hat{\pi}_{h 1}+\frac{n_{h 2}}{n_{h}} \hat{\pi}_{h 2}\right]
$$

with variance given by

$$
\operatorname{Var}\left(\hat{\pi}_{s}\right)=\sum_{h=1}^{L} W_{h}^{2}\left[\frac{n_{h 1}}{n_{h}^{2}}\left\{\frac{\left(1-\pi_{s h}\right)\left(p_{h 1} \pi_{s h}+1-p_{h 1}\right)}{p_{h 1}}\right\}\right.
$$




$$
\left.+\frac{n_{h 2}}{n_{h}^{2}}\left\{\frac{\left(1-\pi_{s h}\right)\left(p_{h 2} \pi_{s h}+1-p_{h 2}\right)}{p_{h 2}}\right\}\right]
$$

where, $\hat{\pi}_{s h 1}=\frac{\hat{\lambda}_{h 1}-\left(1-p_{h 1}\right)}{p_{h 1}}, \hat{\pi}_{s h 2}=\frac{\hat{\lambda}_{h 2}-\left(1-p_{h 2}\right)}{p_{h 2}}$ and $\hat{\pi}_{s h}=\frac{n_{h 1}}{n_{h}} \hat{\pi}_{h 1}+$ $\frac{n_{h 2}}{n_{h}} \hat{\pi}_{h 2}$. If $p_{h 1}=p_{h 2}=p_{h}$ and $n_{h 1}=n_{h 2}=n_{o h}$, the above estimator reduces to

$$
\hat{\pi}_{s}=\sum_{h=1}^{L} W_{h} \hat{\pi}_{s h}=\sum_{h=1}^{L} W_{h} \frac{2 n_{o h}}{n_{h}}\left[\frac{\hat{\lambda}_{h}-\left(1-p_{h}\right)}{p_{h}}\right]
$$

with variance

$$
\begin{aligned}
& \operatorname{Var}\left(\hat{\pi}_{s}\right)=\frac{1}{n}\left[\sum _ { h = 1 } ^ { L } W _ { h } \left\{\pi_{s h}\left(1-\pi_{s h}\right)\right.\right. \\
& \left.\left.+\frac{\left(1-p_{h}\right)\left(1-\pi_{s h}\right)}{p_{h}}\right\}^{\frac{1}{2}}\right]^{2}
\end{aligned}
$$

where, $\hat{\pi}_{s h}=2 \hat{\lambda}_{h}\left[\frac{\hat{\lambda}_{h}-\left(1-p_{h}\right)}{p_{h}}\right]$ for $h=1,2, \cdots, L$.

\section{Methodology}

\subsection{Randomization Procedure}

A simple random sampling with replacement (SRSWR) of $n$ respondents was selected from the population. An individual respondent in the sample of size $n$ was instructed to use the randomization device $(R)$ which consists of a sensitive question (S) card selected with probability $p(p \neq 1$ is a pre-assigned value set by the researcher) and unrelated question (U) card selected with probability $(1-p)$. The paired unrelated or innocuous question has a definite "no"response, for example

(i) S- Are you a member of the insurgence group?

(ii) U- Is this month of February (when research is conducted in the month of September)?

In an applied research, the phrase inside bracket of the unrelated question must be excluded. After the researcher explained the procedures, randomization procedure then followed. Each subject was given a box1 (containing both cards) to select at random a card and tick "yes"or "no"(based on his or her true status, i.e., under the assumption that respondents answer truthfully) and return the ticked card through an opener in the box 2 . Respondents were not placed in close proximity and instructed not to let anyone (the investigator inclusive) see the card he or she had drawn so as to maintain the integrity of the randomized response technique, and keep the individual respondent's privacy or anonymity protected. The probability tree diagram below illustrates the proposed modified-FRRM:

\subsection{Estimation Procedure: Classical Approach}

Let

$$
X_{i}= \begin{cases}0, & \text { if } i^{\text {th }} \text { respondent says no } \\ 1, & \text { if } i^{\text {th }} \text { respondent says yes. }\end{cases}
$$

Elementary probability theory can then be used to get an unbiased estimate of the prevalence $\left(\hat{\pi}_{s}\right)$ of sensitive issues in the population. The procedure followed thus: from Figure 2, the probability of a yes and no response are $\lambda=p \pi_{s}$ and $1-\lambda=$ $p\left(1-\pi_{s}\right)+(1-p)=1-p \pi_{s}$, respectively. Therefore, the likelihood function of $\pi_{s}$ is

$$
L\left(\pi_{s}\right)=\left(\begin{array}{c}
n \\
n_{o}
\end{array}\right)\left[p \pi_{s}\right]^{n_{o}}\left[p\left(1-\pi_{s}\right)+(1-p)\right]^{n-n_{o}} .
$$

where $n_{o}$ is the total number of yes answers in the sample of size $n$ respondents. To estimate $\pi_{s},\left(\begin{array}{c}n \\ n_{0}\end{array}\right)$ in (16) does not contain $\pi_{s}$, as a result, the study considers it to be constant. Hence, the function to maximize reduces to

$$
L\left(\pi_{s}\right)=\left[p \pi_{s}\right]^{n_{o}}\left[p\left(1-\pi_{s}\right)+(1-p)\right]^{n-n_{o}} .
$$

Since logarithm is a monotone function, $\hat{\pi}_{s}$ that maximizes the likelihood function also maximizes its log-likelihood function. Therefore, to facilitate computation, the natural logarithm of (17) yield the log-likelihood function as

$$
l\left(\pi_{s}\right)=n_{o} \ln \left[p \pi_{s}\right]+\left(n-n_{o}\right) \ln \left[p\left(1-\pi_{s}\right)+(1-p)\right] .
$$

Differentiating (18) with respect to $\pi_{s}$, the differential coefficient is

$$
\frac{d}{d \pi_{s}} l\left(\pi_{s}\right)=\frac{n_{o} p}{p \pi_{s}}+\frac{-\left(n-n_{o}\right) p}{p\left(1-\pi_{s}\right)+(1-p)} .
$$

Equating (19) to zero and simplifying the resulting algebraic equation, the maximum likelihood estimator of $\pi_{s}$ which shall be arbitrarily denoted in this study as $\pi_{\text {spropo }}$ is

$$
\hat{\pi}_{\text {spropo }}=\frac{n_{o}}{n p}, \quad p \neq 1 .
$$

\section{Remarks 1}

(i) Putting $p=1$ in either (16) or (20), the proposed MFRRM reduces to the conventional method of direct questioning and the proposed RRT estimator also becomes the traditional measure of proportion. The value of $p=1$ is absolute lack of protection, hence, $p=1$ is impracticable in any randomized response survey. When $0<p<\frac{1}{2}$ or $\frac{1}{2}<p<1$, the respondent gives partially useful but not accurate information to which class (sensitive or not) he or she belongs.

(ii) Like [11], [15] and [36], the proposed estimator $\hat{\pi}_{\text {spropo }}$ is not admissible since the range of $\hat{\pi}_{\text {spropo }}$ is not a subspace of $(0,1)$. In fact, if $\frac{n_{o}}{n}>p$, that is, observed proportion of yes is greater than the pre-assigned randomization parameter, the proposed estimator produce estimate whose value is greater than unity. 


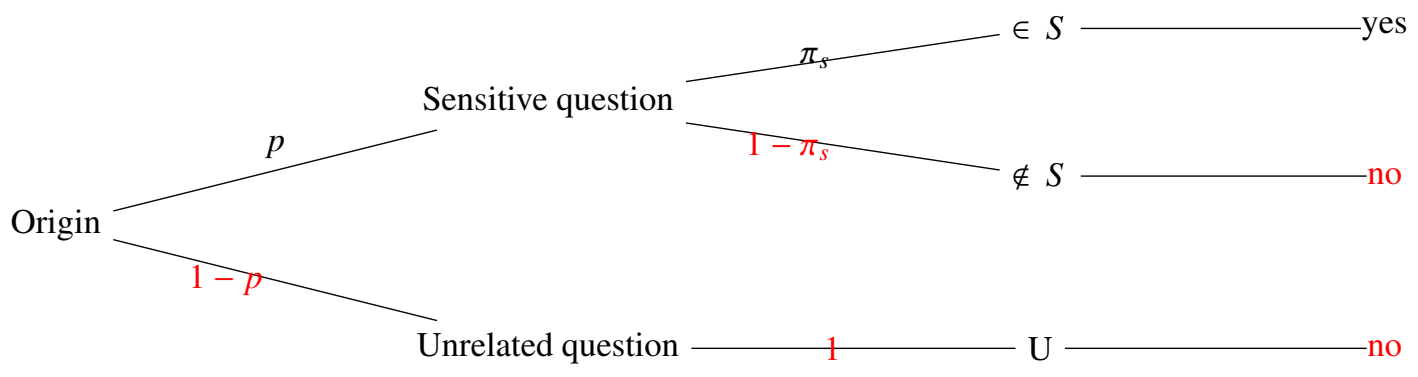

Figure 2: Probability tree diagram of the proposed MFRR model

\subsubsection{Sampling Distribution of the Proposed Estimator $\left(\hat{\pi}_{\text {spropo }}\right)$}

Theorem 3.1 (Unbiasedness of $\hat{\pi}_{\text {spropo }}$ ). The proposed estimator is an unbiased estimator of population proportion of the sensitive attribute under survey.

proof 3.1. $\hat{\pi}_{\text {spropo }}$ is said to be unbiased of the population parameter $\pi_{s}$ if and only if $E\left(\hat{\pi}_{\text {spropo }}\right)=\pi_{s}$. Hence, we take expectation of (20) as follows

$$
\begin{aligned}
E\left(\hat{\pi}_{\text {spropo }}\right) & =E\left[\frac{n_{o}}{n p}\right] \\
& =\frac{1}{n p} \sum_{i=1}^{n}\left[E\left(X_{i}\right)\right]=\frac{1}{n p} n p \pi_{s} \\
& =\pi_{s},
\end{aligned}
$$

which proves the theorem.

Theorem 3.2 (Variance of $\hat{\pi}_{\text {spropo }}$ ). The variance of the proposed estimator is

$$
\operatorname{Var}\left(\hat{\pi}_{\text {spropo }}\right)=\frac{\pi_{s}\left(1-\pi_{s}\right)}{n}+\frac{(1-p) \pi_{s}}{n p} .
$$

proof 3.2. This follows from taking the variance of (20) and considering section 5.5 of [28],

$$
\begin{aligned}
\operatorname{Var}\left(\hat{\pi}_{\text {spropo }}\right) & =\operatorname{Var}\left[\frac{n_{o}}{n p}\right]=\frac{1}{(n p)^{2}} \sum_{i=1}^{n} \operatorname{Var}\left(X_{i}\right) \\
& =\frac{n p \pi_{s}\left[p\left(1-\pi_{s}\right)+(1-p)\right]}{(n p)^{2}} \\
& =\frac{\pi_{s}\left(1-\pi_{s}\right)}{n}+\frac{(1-p) \pi_{s}}{n p} .
\end{aligned}
$$

The sample estimator of $\operatorname{Var}\left(\hat{\pi}_{\text {spropo }}\right)$ is obtained by substituting (20) in the preceding equation to have

$$
\hat{V} \operatorname{ar}\left(\hat{\pi}_{\text {spropo }}\right)=\frac{\hat{\pi}_{s}\left(1-\hat{\pi}_{s}\right)}{n}+\frac{(1-p) \hat{\pi}_{s}}{n p} .
$$

Remark 2: Equation (23) consists of two parts. The first is the variance of the population proportion of the sensitive attribute if all the respondents are willing to imbibe direct questioning approach. The second part is injected due to use of randomizer. Putting $\pi_{u}=0$ in equation (4) and (5), the results coincident with the proposed estimator in (20) and (22), respectively.

\subsubsection{Stratified Sampling Strategy for the Proposed MFRRM}

If in the proposed model, the population $(P)$ is partitioned (using a suitable or appropriate stratification factor) into $L$ strata, and a sample $n_{h}(h=1,2, \cdots, L)$ is selected by simple random sampling with replacement in each stratum such that $n=\sum_{h=1}^{L} n_{h}$. To get the full benefit from stratification, the study assume that $N_{h}$ (the number of units in each stratum) is known. Following the same randomization procedure of the proposed (SRSWR) model, a respondent belonging to the sample in different strata will perform different randomization devices, each having different preassigned probabilities $p_{h}$. Under the assumption that these respondents "yes"or "no"-reports are made truthfully and $p_{h} \neq 1$ is set by the researcher. The probability of a "yes" and "no" response from stratum $h$ are $\operatorname{Pr}\left(X_{i h}=1\right)=\lambda_{h}=p_{h} \pi_{s h}$ and $\operatorname{Pr}\left(X_{i h}=0\right)=1-\lambda_{h}=p_{h}\left(1-\pi_{s h}\right)+\left(1-p_{h}\right)$ for $h=1,2, \cdots, L$ respectively.

Suppose $n_{\text {oh }}$ report "yes" and $\left(n_{h}-n_{\text {oh }}\right)$ report "no"to the sensitive question in stratum $h$, the likelihood function of the sample in stratum $h$ is

$$
L\left(\pi_{s h}\right)=\left[p_{h} \pi_{s h}\right]^{n_{o h}}\left[p_{h}\left(1-\pi_{s h}\right)+\left(1-p_{h}\right)\right]^{\left(n_{h}-n_{o h}\right)}
$$

For computational convenience, the natural logarithm of the likelihood function is

$$
l\left(\pi_{s h}\right)=n_{o h} \ln \left[p_{h} \pi_{s h}\right]+\left(n_{h}-n_{o h}\right) \ln \left[p_{h}\left(1-\pi_{s h}\right)+\left(1-p_{h}\right)\right]
$$

To obtain the maximum likelihood estimator of $\pi_{s h}$, the study differentiate (25) with respect to $\pi_{s h}$ and equating the derivative to zero to get

$$
0=\frac{n_{o h} p_{h}}{p_{h} \pi_{s h}}-\frac{\left(n_{h}-n_{o h}\right) p_{h}}{p_{h}\left(1-\pi_{s h}\right)+\left(1-p_{h}\right)}
$$

solving the resulting equation (26) for $\pi_{s h}$, the unbiased estimators in terms of the responses of the respondent in stratum $h$ is given by

$$
\hat{\pi}_{s h}=\frac{n_{o h}}{n_{h} p_{h}}=\frac{\hat{\lambda}_{h}}{p_{h}}
$$

where $\hat{\lambda}_{h}=$ proportion of "yes"-answers in stratum $h$ and $\hat{\pi}_{s h}$ is an unbiased estimate for $\pi_{s h}$.

Variance of $\hat{\pi}_{s h}$ : The variance of $\hat{\pi}_{s h}$ is obtained by taking vari- 
ance of (27) as follows:

$$
\begin{aligned}
\operatorname{Var}\left(\hat{\pi}_{s h}\right) & =\operatorname{Var}\left[\frac{n_{o h}}{n_{h} p_{h}}\right]=\frac{1}{\left(n_{h} p_{h}\right)^{2}} \operatorname{Var}\left(n_{o h}\right) \\
& =\frac{n_{h} p_{h} \pi_{s h}\left[p_{h}\left(1-\pi_{s h}\right)+\left(1-p_{h}\right)\right]}{\left(n_{h} p_{h}\right)^{2}} \\
& =\frac{p_{h} \pi_{s h}\left(1-\pi_{s h}\right)}{n_{h} p_{h}}+\frac{\pi_{s h}\left(1-p_{h}\right)}{n_{h} p_{h}} .
\end{aligned}
$$

Hence,

$$
\operatorname{Var}\left(\hat{\pi}_{s h}\right)=\frac{\pi_{s h}\left(1-\pi_{s h}\right)}{n_{h}}+\frac{\pi_{s h}\left(1-p_{h}\right)}{n_{h} p_{h}} .
$$

Since selections in different strata are made independently, the maximum likelihood estimate of $\pi_{s}$ is easily shown to be

$$
\hat{\pi}_{\text {stpropo }}=\sum_{h=1}^{L} W_{h} \hat{\pi}_{s h}=\sum_{h=1}^{L} W_{h} \frac{\hat{\lambda}_{h}}{p_{h}}=\frac{1}{N} \sum_{h=1}^{L} N_{h} \frac{n_{o h}}{n_{h} p_{h}}
$$

where $N$ and $N_{h}$ denote the number of subjects in the whole population and in the stratum $h$, respectively. $W_{h}$ (stratum weight) $=\frac{N_{h}}{N}$, for $h=1,2, \cdots, L$ so that $\sum_{h=1}^{L} W_{h}=1$.

\subsubsection{Sampling Distribution of the Proposed Stratified Estimator $\hat{\pi}_{\text {stpropo }}$}

Theorem 3.3 (Unbiasedness of $\hat{\pi}_{\text {stpropo }}$ ). The proposed stratified estimator is an unbiased estimator of population proportion of the sensitive attribute under study.

proof 3.3. $\hat{\pi}_{\text {stpropo }}$ is said to be unbiased of the population parameter $\pi_{s}$ if $E\left(\hat{\pi}_{\text {stpropo }}\right)=\pi_{s}$. Hence, we take expectation of (29) and provided that $\hat{\pi}_{s h}$ is unbiased for $\pi_{s h}$,

$$
E\left(\hat{\pi}_{\text {stpropo }}\right)=E\left(\sum_{i=1}^{L} W_{h} \hat{\pi}_{s h}\right)=\sum_{h=1}^{L} W_{h} E\left(\hat{\pi}_{s h}\right)=\sum_{h=1}^{L} W_{h} \pi_{s h}=\pi_{s}
$$

Theorem 3.4 (Variance of $\hat{\pi}_{\text {stpropo }}$ ). The variance of $\hat{\pi}_{\text {stpropo }}$ is

$$
\operatorname{Var}\left(\hat{\pi}_{\text {stpropo }}\right)=\frac{1}{n}\left[\sum_{h=1}^{L} W_{h}\left\{\pi_{s h}\left(1-\pi_{s h}\right)+\frac{\pi_{s h}\left(1-p_{h}\right)}{p_{h}}\right\}^{\frac{1}{2}}\right]^{2} .
$$

proof 3.4. This follows from taking the variance of (29) and from corollary 1 in section 5.9 of [28], since each unbiased estimator $\hat{\pi}_{\text {sh }}$ has its own variance, the variance of $\hat{\pi}_{\text {stpropo }}$ is

$$
\operatorname{Var}\left(\hat{\pi}_{\text {stpropo }}\right)=\operatorname{Var}\left(\sum_{h=1}^{L} W_{h} \hat{\pi}_{s h}\right)=\sum_{h=1}^{L} W_{h}^{2} \operatorname{Var}\left(\hat{\pi}_{s h}\right) .
$$

Substituting (28) into (32), we have

$$
\operatorname{Var}\left(\hat{\pi}_{\text {stpropo }}\right)=\sum_{h=1}^{L} \frac{W_{h}^{2}}{n_{h}}\left[\pi_{s h}\left(1-\pi_{s h}\right)+\frac{\pi_{s h}\left(1-p_{h}\right)}{p_{h}}\right]
$$

Recall, simple random sampling scheme asserts that $\operatorname{Var}(\bar{y})=$ $(1-f) \frac{S_{y}^{2}}{n}$. Similarly,

$$
\operatorname{Var}\left(\hat{\pi}_{s h}\right)=\left(1-f_{h}\right) \frac{S_{\pi_{s h}}^{2}}{n_{h}}=\left(1-\frac{n_{h}}{n}\right) \frac{S_{\pi_{s h}}^{2}}{n_{h}} .
$$

[28] established that when sampling with replacement, the sampling fraction is ignorable as $n \longrightarrow N$ relative to $n_{h}, \frac{n_{h}}{n} \longrightarrow 0$ and $1-f_{h}=1-\frac{n_{h}}{n} \longrightarrow 1$. Therefore, equation (34) reduces to

$$
\operatorname{Var}\left(\hat{\pi}_{s h}\right)=\frac{S_{\pi_{s h}}^{2}}{n_{h}}
$$

making $S_{\pi_{s h}}$ subject of relation from equation (35) and substituting equation (28) into the resulting expression produces

$$
S_{\pi_{s h}}=\left[\pi_{s h}\left(1-\pi_{s h}\right)+\frac{\left(1-p_{h}\right) \pi_{s h}}{p_{h}}\right]^{\frac{1}{2}} .
$$

However, by optimum allocation the sample sizes are defined to minimize variance with a given cost. For fixed cost, by the Cauchy-Schwarz inequality the sample size $n_{h}$ to minimize $\operatorname{Var}\left(\hat{\pi}_{\text {stpropo }}\right)$ is given by

$$
n_{h}=\frac{n W_{h} S_{\pi_{s h}}}{\sum_{h=1}^{L} W_{h} S_{\pi_{s h}}}
$$

The optimal allocation of $n$ to $n_{1}, n_{2}, \cdots, n_{L-1}, n_{L}$ to derive the minimum variance of $\hat{\pi}_{s}$ subject to $n=\sum_{h=1}^{L} n_{h}$ is obtained by substituting (36) into (37) to give

$$
n_{h}=\frac{n W_{h}\left[\pi_{s h}\left(1-\pi_{s h}\right)+\frac{\pi_{s h}\left(1-p_{h}\right)}{p_{h}}\right]^{\frac{1}{2}}}{\sum_{h=1}^{L} W_{h}\left[\pi_{s h}\left(1-\pi_{s h}\right)+\frac{\pi_{s h}\left(1-p_{h}\right)}{p_{h}}\right]^{\frac{1}{2}}} .
$$

The minimal variance of $\hat{\pi}_{\text {stpropo }}$ is then obtained by substituting (38) into (33), so that

$$
\begin{aligned}
& \operatorname{Var}\left(\hat{\pi}_{\text {stpropo }}\right)=\sum_{h=1}^{L} W_{h}^{2}\left\{\pi_{s h}\left(1-\pi_{s h}\right)+\frac{\pi_{s h}\left(1-p_{h}\right)}{p_{h}}\right\} \\
& \div\left[\frac{n W_{h}\left\{\pi_{s h}\left(1-\pi_{s h}\right)+\frac{\pi_{s h}\left(1-p_{h}\right)}{p_{h}}\right\}^{\frac{1}{2}}}{\sum_{h=1}^{L}\left\{\pi_{s h}\left(1-\pi_{s h}\right)+\frac{\pi_{s h}\left(1-p_{h}\right)}{p_{h}}\right\}^{\frac{1}{2}}}\right] \\
& \operatorname{Var}\left(\hat{\pi}_{\text {stpropo }}\right)=\sum_{h=1}^{L} W_{h}^{2}\left\{\pi_{s h}\left(1-\pi_{s h}\right)+\frac{\pi_{s h}\left(1-p_{h}\right)}{p_{h}}\right\} \\
& \times \frac{\sum_{h=1}^{L}\left\{\pi_{s h}\left(1-\pi_{s h}\right)+\frac{\pi_{s h}\left(1-p_{h}\right)}{p_{h}}\right\}^{\frac{1}{2}}}{n W_{h}\left\{\pi_{s h}\left(1-\pi_{s h}\right)+\frac{\pi_{s h}\left(1-p_{h}\right)}{p_{h}}\right\}^{\frac{1}{2}}}
\end{aligned}
$$

Further simplification of (40) yields

$$
\operatorname{Var}\left(\hat{\pi}_{\text {stpropo }}\right)=\frac{1}{n}\left[\sum_{h=1}^{L} W_{h}\left\{\pi_{s h}\left(1-\pi_{s h}\right)+\frac{\pi_{s h}\left(1-p_{h}\right)}{p_{h}}\right\}^{\frac{1}{2}}\right]^{2}
$$

The unbiased minimal variance of $\hat{\pi}_{\text {stpropo }}$ follows on replacing $n$ by $(n-1)$ in equation (31) to get 


$$
\begin{aligned}
\hat{\operatorname{Var}}\left(\hat{\pi}_{\text {stpropo }}\right)= & \frac{1}{n-1}\left[\sum _ { h = 1 } ^ { L } W _ { h } \left\{\pi_{s h}\left(1-\pi_{s h}\right)\right.\right. \\
& \left.\left.+\frac{\pi_{s h}\left(1-p_{h}\right)}{p_{h}}\right\}^{\frac{1}{2}}\right]^{2}
\end{aligned}
$$

Although, if $n$ is large, the difference between equation (31) and (41) is negligible. If $p_{h}=p$ for $h=1,2, \cdots, L,(31)$ reduces to

$$
\begin{gathered}
\operatorname{Var}\left(\hat{\pi}_{\text {stpropo }}\right)=\frac{1}{n}\left[\sum _ { h = 1 } ^ { L } W _ { h } \left\{\pi_{s h}\left(1-\pi_{s h}\right)\right.\right. \\
\left.\left.+\frac{\pi_{s h}(1-p)}{p}\right\}^{\frac{1}{2}}\right]^{2}
\end{gathered}
$$

To facilitate computation of $\operatorname{Var}\left(\hat{\pi}_{\text {stpropo }}\right)$, let $\left\{\pi_{s h}\left(1-\pi_{s h}\right)\right.$ $\left.+\frac{\pi_{s h}\left(1-p_{h}\right)}{p_{h}}\right\}^{\frac{1}{2}}$ be replaced by $\phi_{s h}$ in equation (31) so that

$$
\operatorname{Var}\left(\hat{\pi}_{\text {stpropo }}\right)=\frac{1}{n}\left[\sum_{h=1}^{L} W_{h} \phi_{s h}\right]^{2}
$$

\subsection{Efficiency Comparison}

The efficiency of the proposed model with Warner's original model, Boruch's FRRM and Mangat's improved two step procedure is judged by Mean Square Error (MSE) criterion. It should be noted that since the existing and the proposed estimators are unbiased, then the criterion for judging the performance of the proposed estimator is now limited to variance comparison.

\subsubsection{Efficiency comparison with Warner's (1965) Original RRM}

In this study, we arbitrarily denote variance under Warner design as $\operatorname{Var}\left(\hat{\pi}_{\mathrm{sw}}\right)$ and the proposed model as $\operatorname{Var}\left(\hat{\pi}_{\text {spropo }}\right)$. Recall from equations (3) and (22)

$$
\operatorname{Var}\left(\hat{\pi}_{\mathrm{s} \text { warner }}\right)=\frac{\pi_{s}\left(1-\pi_{s}\right)}{n}+\frac{p(1-p)}{n(2 p-1)^{2}}
$$

and

$$
\operatorname{Var}\left(\hat{\pi}_{\mathrm{spropo}}\right)=\frac{\pi_{s}\left(1-\pi_{s}\right)}{n}+\frac{(1-p) \pi_{s}}{n p}
$$

The proposed modified FRRM is more efficient than Warner's $\mathrm{RRM}$ if $\operatorname{Var}\left(\hat{\pi}_{\mathrm{s} \text { warner }}\right)-\operatorname{Var}\left(\hat{\pi}_{\mathrm{s} \text { propo }}\right) \geq 0$. That is,

$$
\left\{\frac{\pi_{s}\left(1-\pi_{s}\right)}{n}+\frac{p(1-p)}{n(2 p-1)^{2}}\right\}-\left\{\frac{\pi_{s}\left(1-\pi_{s}\right)}{n}+\frac{(1-p) \pi_{s}}{n p}\right\} \geq 0
$$

This implies that

$$
p^{2}-(2 p-1)^{2} \pi_{s} \geq 0 \forall p \in[0,1]
$$

The inequality (44) always holds. Therefore, the proposed model is more efficient than [7] original model.

\subsubsection{Efficiency Comparison with Boruch (1971) Original FRRM}

Recall from equation (5), the Boruch's variance estimator of $\pi_{s}$ which is subjectively denoted here as $\operatorname{Var}\left(\hat{\pi}_{\text {s.boruch }}\right)$ is

$$
\begin{aligned}
\operatorname{Var}\left(\hat{\pi}_{\text {s.boruch }}\right)= & \frac{\pi_{s}\left(1-\pi_{s}\right)}{n}+\frac{(1-p)}{n p}\left[\pi_{s}\left(1-\pi_{u}\right)+\pi_{u}\left(1-\pi_{s}\right)\right. \\
& \left.+\frac{(1-p) \pi_{u}\left(1-\pi_{u}\right)}{p}\right]
\end{aligned}
$$

The proposed MFRRM is more efficient than Boruch's FRRM if $\operatorname{Var}\left(\hat{\pi}_{\text {s.boruch }}\right)-\operatorname{Var}\left(\hat{\pi}_{\text {spropo }}\right) \geq 0$. That is,

$$
\begin{aligned}
& \frac{\pi_{s}\left(1-\pi_{s}\right)}{n}+\frac{(1-p)}{n p}\left[\pi_{s}\left(1-\pi_{u}\right)+\pi_{u}\left(1-\pi_{s}\right)\right. \\
& \left.+\frac{(1-p) \pi_{u}\left(1-\pi_{u}\right)}{p}\right] \\
& -\left\{\frac{\pi_{s}\left(1-\pi_{s}\right)}{n}+\frac{(1-p) \pi_{s}}{n p}\right\}
\end{aligned}
$$

which implies

$$
\pi_{s}\left(1-\pi_{u}\right)+\pi_{u}\left(1-\pi_{s}\right)+\frac{(1-p) \pi_{u}\left(1-\pi_{u}\right)}{p}-\pi_{s}
$$

and further simplification gives

$$
\pi_{s} \leq \frac{1-\pi_{u}(1-p)}{2 p} .
$$

If the condition in (47) is satisfied then the proposed MFFRM is more efficient than [12] original FRRM.

\subsubsection{Efficiency Comparison with Mangat's (1994) Improved Two-Step Procedure}

From equation (7), the variance of $\pi_{s}$ under Mangat design is

$$
\operatorname{Var}\left(\hat{\pi}_{\mathrm{s}}\right)=\frac{\pi_{s}\left(1-\pi_{s}\right)}{n}+\frac{(1-p)\left(1-\pi_{s}\right)}{n p}
$$

The proposed MFRRM is more efficient than Mangat's improved two-step procedure if $\operatorname{Var}\left(\hat{\pi}_{\mathrm{s} \text { mangat }}\right)-\operatorname{Var}\left(\hat{\pi}_{\mathrm{s} \text { propo }}\right) \geq 0$. That is,

$$
\left\{\frac{\pi_{s}\left(1-\pi_{s}\right)}{n}+\frac{(1-p)\left(1-\pi_{s}\right)}{n p}\right\}-\left\{\frac{\pi_{s}\left(1-\pi_{s}\right)}{n}+\frac{(1-p) \pi_{s}}{n p}\right\} \geq 0
$$

After simplification,

$$
1-2 \pi_{s} \geq 0, \Longrightarrow \pi_{s} \leq \frac{1}{2}
$$

The inequality (48) holds $\forall \pi_{s} \leq \frac{1}{2}$. Hence, the proposed MFRRM is more efficient than Mangat's improved two step procedure if and only if $\pi_{s} \leq \frac{1}{2}$. 


\subsubsection{Efficiency Comparison with Kim and Warde Strati- fore, \\ fied Model}

Recall, variance of $\pi_{s}$ under Kim and Warde literally represented as $\operatorname{Var}\left(\hat{\pi}_{\text {skw }}\right)$ and proposed stratified MFRRM are

$$
\begin{aligned}
\operatorname{Var}\left(\hat{\pi}_{\text {skw }}\right) & =\frac{1}{n}\left[\sum_{h=1}^{L} W_{h}\left\{\pi_{s h}\left(1-\pi_{s h}\right)+\frac{p_{h}\left(1-p_{h}\right)}{\left(2 p_{h}-1\right)^{2}}\right\}^{\frac{1}{2}}\right]^{2} \text { and } \\
\operatorname{Var}\left(\hat{\pi}_{\text {stpropo }}\right) & =\frac{1}{n}\left[\sum_{h=1}^{L} W_{h}\left\{\pi_{s h}\left(1-\pi_{s h}\right)+\frac{\pi_{s h}\left(1-p_{h}\right)}{p_{h}}\right\}^{\frac{1}{2}}\right]^{2},
\end{aligned}
$$

respectively. The proposed stratified MFRRM is more efficient than the [16] stratified model if the relative efficiency $(R E)=$ $\frac{\operatorname{Var}\left(\hat{(}_{\text {skw }}\right)}{\operatorname{Var}\left(\hat{\mathrm{s}}_{\text {stropo }}\right)} \geq 1$. That is, $\operatorname{Var}\left(\hat{\pi}_{\text {skw }}\right)-\operatorname{Var}\left(\hat{\pi}_{\text {stpropo }}\right) \geq 0$. Using this condition,

$$
\begin{aligned}
& \frac{1}{n}\left[\sum_{h=1}^{L} W_{h}\left\{\pi_{s h}\left(1-\pi_{s h}\right)+\frac{p_{h}\left(1-p_{h}\right)}{\left(2 p_{h}-1\right)^{2}}\right\}^{\frac{1}{2}}\right]^{2} \\
& -\frac{1}{n}\left[\sum_{h=1}^{L} W_{h}\left\{\pi_{s h}\left(1-\pi_{s h}\right)+\frac{\pi_{s h}\left(1-p_{h}\right)}{p_{h}}\right\}^{\frac{1}{2}}\right]^{2} \geq 0 .
\end{aligned}
$$

The above inequality is true if for each stratum $h, h=1,2, \cdots, L$ we have

$\left[\pi_{s h}\left(1-\pi_{s h}\right)+\frac{p_{h}\left(1-p_{h}\right)}{\left(2 p_{h}-1\right)^{2}}\right]^{\frac{1}{2}}-\left[\pi_{s h}\left(1-\pi_{s h}\right)+\frac{\pi_{s h}\left(1-p_{h}\right)}{p_{h}}\right]^{\frac{1}{2}} \geq 0$

which implies

$$
\frac{p_{h}\left(1-p_{h}\right)}{\left(2 p_{h}-1\right)^{2}}-\frac{\pi_{s h}\left(1-p_{h}\right)}{p_{h}} \geq 0
$$

Multiplying the preceding inequality throughout by $\frac{p_{h}\left(2 p_{h}-1\right)^{2}}{1-p_{h}}$ gives

$$
p_{h}^{2}-\left(2 p_{h}-1\right)^{2} \pi_{s h} \geq 0 \quad \forall p_{h} \in(0,1) \text { and } \forall \pi_{s h} \in(0,1)(50)
$$

The LHS of (50) is always non-negative, hence the proposed stratified MFRRM is more efficient than [16] stratified randomized response model.

\subsubsection{Efficiency Comparison With Usman and Oshungade Mixed Stratified RRM}

[17] invented Mixed Stratified RRM for HIV sero-prevalence survey with $\operatorname{Var}\left(\hat{\pi}_{s}\right)$ as

$$
\operatorname{Var}\left(\hat{\pi}_{\mathrm{s}}\right)=\frac{1}{n}\left[\sum_{h=1}^{L} W_{h}\left\{\pi_{s h}\left(1-\pi_{s h}\right)+\frac{\left(1-p_{h}\right)\left(1-\pi_{s h}\right)}{p_{h}}\right\}^{\frac{1}{2}}\right]^{2} .
$$

The proposed stratified Modified FRRM is more efficient than the [17] mixed stratified RRM if the relative efficiency $(R E)=$ $\frac{\operatorname{Var}\left(\hat{\pi}_{\text {susman }}\right)}{\operatorname{Var}\left(\hat{\pi}_{\text {sppropo }}\right)} \geq 1$. That is, $\operatorname{Var}\left(\hat{\pi}_{\mathrm{s} \text { usman }}\right)-\operatorname{Var}\left(\hat{\pi}_{\text {stpropo }}\right) \geq 0$. There-

$$
\begin{aligned}
& \frac{1}{n}\left[\sum_{h=1}^{L} W_{h}\left\{\pi_{s h}\left(1-\pi_{s h}\right)+\frac{\left(1-p_{h}\right)\left(1-\pi_{s h}\right)}{p_{h}}\right\}^{\frac{1}{2}}\right]^{2} \\
& -\frac{1}{n}\left[\sum_{h=1}^{L} W_{h}\left\{\pi_{s h}\left(1-\pi_{s h}\right)+\frac{\pi_{s h}\left(1-p_{h}\right)}{p_{h}}\right\}^{\frac{1}{2}}\right]^{2} \geq(51)
\end{aligned}
$$

The inequality (51) holds if for each stratum $h(h=1,2, \cdots, L)$ we have

$$
\begin{aligned}
& \left\{\pi_{s h}\left(1-\pi_{s h}\right)+\frac{\left(1-p_{h}\right)\left(1-\pi_{s h}\right)}{p_{h}}\right\}^{\frac{1}{2}} \\
& -\left\{\pi_{s h}\left(1-\pi_{s h}\right)+\frac{\pi_{s h}\left(1-p_{h}\right)}{p_{h}}\right\}^{\frac{1}{2}} \geq 0
\end{aligned}
$$

which reduces to

$$
\frac{\left(1-p_{h}\right)\left(1-\pi_{s h}\right)}{p_{h}}-\frac{\pi_{s h}\left(1-p_{h}\right)}{p_{h}} \geq 0 .
$$

Multiplying the above inequality (53) through by $\frac{p_{h}}{1-p_{h}}$ yields

$$
1-2 \pi_{s h} \geq 0 .
$$

The inequality (54) holds $\forall \pi_{s h} \leq \frac{1}{2}$. That is, the proposed model is more efficient than the [17] mixed stratified RR model if $\pi_{s h} \leq \frac{1}{2}$ for $h=1,2, \cdots, L$.

\subsubsection{Cost and Efficiency of Stratification}

It is essential to think about more than two or three strata cases in terms of efficiency. [28] showed that the variance for the mean of a stratified random sample decreases as the number of strata increases. So, this section explore the behaviour of $\operatorname{Var}\left(\hat{\pi}_{\text {stpropo }}\right)$ as the number of strata increases. Suppose $L$ strata of equal sizes are created such that $W_{h}=\frac{1}{L}$, substituting $W_{h}=\frac{1}{L}$ in equation (31) produces

$$
\operatorname{Var}\left(\hat{\pi}_{\text {stpropo }}\right)=\frac{1}{n L^{2}}\left[\sum_{h=1}^{L}\left\{\pi_{s h}\left(1-\pi_{s h}\right)+\frac{\pi_{s h}\left(1-p_{h}\right)}{p_{h}}\right\}^{\frac{1}{2}}\right]^{2}
$$

Let $f(L)=\frac{1}{L^{2}}\left[\sum_{h=1}^{L}\left\{\pi_{s h}\left(1-\pi_{s h}\right)+\frac{\pi_{s h}\left(1-p_{h}\right)}{p_{h}}\right\}^{\frac{1}{2}}\right]^{2}$ where $L$ is a positive integer, We want to show that $f(L)-f(L+1) \geq 0$ for $L\left(\pi_{s h}, p_{h}\right)=\left\{\pi_{s h}\left(1-\pi_{s h}\right)+\frac{\pi_{s h}\left(1-p_{h}\right)}{p_{h}}\right\}^{\frac{1}{2}}$.

$$
\begin{aligned}
f(L)-f(L+1) & =\frac{1}{L^{2}}\left[\sum_{h=1}^{L} L\left(\hat{\pi}_{s h}, p_{h}\right)\right]^{2}-\frac{1}{(L+1)^{2}}\left[\sum_{h=1}^{L+1} L\left(\hat{\pi}_{s h}, p_{h}\right)\right]^{2} \\
& =\left[\left(\frac{1}{L} \sum_{h=1}^{L} L\left(\hat{\pi}_{s h}, p_{h}\right)\right)\right]^{2}-\left[\left(\frac{1}{L+1} \sum_{h=1}^{L+1} L\left(\hat{\pi}_{s h}, p_{h}\right)\right)\right]^{2}
\end{aligned}
$$




$$
\begin{aligned}
& =\left[\frac{1}{L}\left(\sum_{h=1}^{L} L\left(\hat{\pi}_{s h}, p_{h}\right)\right)+\frac{1}{L+1}\left(\sum_{h=1}^{L+1} L\left(\hat{\pi}_{s h}, p_{h}\right)\right)\right] \times \\
& {\left[\frac{1}{L}\left(\sum_{h=1}^{L} L\left(\hat{\pi}_{s h}, p_{h}\right)\right)-\frac{1}{L+1}\left(\sum_{h=1}^{L+1} L\left(\hat{\pi}_{s h}, p_{h}\right)\right)\right] .}
\end{aligned}
$$

As the number of strata increases, it may be possible to divide a heterogeneous population into sub-populations, each of which is more homogeneous. So we may get

$$
\left[\frac{1}{L}\left(\sum_{h=1}^{L} L\left(\hat{\pi}_{s h}, p_{h}\right)\right)-\frac{1}{L+1}\left(\sum_{h=1}^{L+1} L\left(\hat{\pi}_{s h}, p_{h}\right)\right)\right] \geq 0 .
$$

By this assumption, $f(L)$ is a monotone decreasing function of $L$. Therefore, the variance of the proposed estimator gets smaller as the number of strata increases.

\subsection{Bayesian Estimation Approach}

In making generalization, conclusion or prediction about unknown population parameter(s) the trend is to distinguish between classical method of estimating the population parameter(s) whereby inferences are based strictly on information obtained from a random sample and a Bayesian one which utilizes prior subjective knowledge about the probability distribution in conjunction with the information provided by the sample data. [29] and [30] estimated Warner's RRM using Bayesian approach with binomial likelihood and beta prior as

$$
\hat{\pi}_{\mathrm{bw}}=\frac{\frac{\left(a+n_{o}\right)}{(a+b+n)}-(1-p)}{(2 p-1)}
$$

with posterior variance defined as

$$
\operatorname{Var}\left(\hat{\pi}_{\mathrm{bw}}\right)=\frac{n \pi_{s}\left(1-\pi_{s}\right)}{(a+b+n)^{2}}+\frac{n p(1-p)}{(2 p-1)^{2}(a+b+n)^{2}} .
$$

Similarly, the model for the proposed sensitive random variable $X$ conditional on unknown parameter $\lambda$ popularly called likelihood function is the density function $f(x \mid \lambda)=L(\lambda)$ given as

$$
\begin{aligned}
L(\lambda) & =f\left(n_{o} \mid \lambda\right)=\left(\begin{array}{c}
n \\
n_{o}
\end{array}\right) \lambda^{n_{o}}(1-\lambda)^{n-n_{o}} \\
& =\left(\begin{array}{c}
n \\
n_{o}
\end{array}\right)\left(p \pi_{s}\right)^{n_{o}}\left[p\left(1-\pi_{s}\right)+(1-p)\right]^{n-n_{o}} .
\end{aligned}
$$

The parameter $\lambda=p \pi_{s}$ is considered a random variable which has a distribution $g(\lambda)$ called the prior distribution otherwise known as prior predictive which describes uncertainty about the parameter before data are observed. A more convenient family of densities for a proportion $\lambda$ is the beta with kernel proportional to $g(\lambda) \propto \lambda^{a-1}(1-\lambda)^{b-1}, 0<\lambda<1$. Hence,

$$
\begin{aligned}
g(\lambda)= & \frac{1}{\beta(a, b)} \lambda^{a-1}(1-\lambda)^{b-1} \\
= & \frac{1}{\beta(a, b)}\left(p \pi_{s}\right)^{a-1}\left[p\left(1-\pi_{s}\right)+(1-p)\right]^{b-1}, \\
& 0<\lambda<1
\end{aligned}
$$

where the hyper-parameters $a$ and $b$ are chosen to reflect the user's prior beliefs about $\lambda$. Our goal is to start with this prior information and update it using the data to make the best possible estimator of $\lambda$. Multiplying this beta prior with the likelihood function gives the joint density function $h\left(n_{o}, \lambda\right)$ as

$$
\begin{aligned}
h\left(n_{o}, \lambda\right) & =f\left(n_{o} \mid \lambda\right) g(\lambda) \\
& =\frac{\left(\begin{array}{c}
n \\
n_{o}
\end{array}\right)}{\beta(a, b)}\left(p \pi_{s}\right)^{a+n_{o}-1}\left[p\left(1-\pi_{s}\right)+(1-p)\right]^{b+n-n}(61)
\end{aligned}
$$

The marginal distribution $\left(m\left(n_{o}\right)\right)$ can be obtained by integrating out parameter $\lambda$ from the joint distribution as

$$
\begin{aligned}
m\left(n_{o}\right) & =\int_{\ominus} h\left(n_{o}, \lambda\right) d \lambda=\int_{\odot} f\left(n_{o} \mid \lambda\right) g(\lambda) d \lambda \\
& =\int_{0}^{1} \frac{\left(\begin{array}{c}
n \\
n_{o}
\end{array}\right)}{\beta(a, b)}\left(p \pi_{s}\right)^{a+n_{o}-1}\left[p\left(1-\pi_{s}\right)+(1-p)\right]^{b+n-n_{o}-1} d\left(p \pi_{s}\right) \\
& =\int_{0}^{1} \frac{\left(\begin{array}{c}
n \\
n_{o}
\end{array}\right)}{\beta(a, b)}\left(p \pi_{s}\right)^{a+n_{o}-1}\left(1-p \pi_{s}\right)^{b+n-n_{o}-1} d\left(p \pi_{s}\right) .
\end{aligned}
$$

Therefore,

$$
m\left(n_{o}\right)=\frac{\left(\begin{array}{c}
n \\
n_{o}
\end{array}\right)}{\beta(a, b)} \beta\left(a+n_{o}, b+n-n_{o}\right) .
$$

Combining this beta prior with the likelihood function, one can find $f\left(\lambda \mid n_{o}\right)$, called the posterior distribution/density for $\lambda$ given $X=n_{o}$ as

$$
\begin{aligned}
f\left(\lambda \mid n_{o}\right) & =\frac{f\left(n_{o} \mid \lambda\right) g(\lambda)}{\int_{\ominus} f\left(n_{o} \mid \lambda\right) g(\lambda) d \lambda}=\frac{h\left(n_{o}, \lambda\right)}{m\left(n_{o}\right)} \\
& =\frac{\left(\begin{array}{c}
n \\
n_{o}
\end{array}\right)}{\beta(a, b)}\left(p \pi_{s}\right)^{a+n_{o}-1}\left[p\left(1-\pi_{s}\right)+(1-p)\right]^{b+n-n_{o}-1} \\
& \times \frac{\beta(a, b)}{\left(\begin{array}{c}
n \\
n_{o}
\end{array}\right) \beta\left(a+n_{o}, b+n-n_{o}\right)}
\end{aligned}
$$

which gives

$$
\begin{aligned}
f\left(\lambda \mid n_{o}\right) & =\frac{\left(p \pi_{s}\right)^{a+n_{o}-1}\left(1-p \pi_{s}\right)^{b+n-n_{o}-1}}{\beta\left(a+n_{o}, b+n-n_{o}\right)} \\
& =\frac{(\lambda)^{a+n_{o}-1}(1-\lambda)^{b+n-n_{o}-1}}{\beta\left(a+n_{o}, b+n-n_{o}\right)} .
\end{aligned}
$$

Like $g(\lambda), f\left(\lambda \mid n_{o}\right)$ also follows beta-distribution with updated parameters $a+n_{o}$ and $b+n-n_{o}$. This is an example of a conjugate analysis where the prior and posterior densities have the same functional form. The prior $g(\lambda)$ is said to be a conjugate with respect to $f\left(n_{o} \mid \lambda\right)[31,32]$.

The Bayes estimator of $h(\lambda)$ under the squared error loss is a ratio of integrals

$$
\hat{h \lambda}=\int_{\ominus} h(\lambda) \pi\left(\lambda \mid n_{o}\right) d \lambda=\frac{\int_{\ominus} h(\lambda) f\left(n_{o} \mid \lambda\right) g(\lambda) d \lambda}{\int_{\ominus} f\left(n_{o} \mid \lambda\right) g(\lambda) d \lambda}
$$


Following from (64) above, the posterior mean $\hat{\lambda}$ is

$$
\begin{aligned}
\hat{\lambda} & =\int_{0}^{1} \lambda f\left(\lambda \mid n_{o}\right) d \lambda=\int_{0}^{1} \lambda \frac{\lambda^{\left(a+n_{o}\right)-1}(1-\lambda)^{\left(b+n-n_{o}\right)-1}}{\beta\left[\left(a+n_{o}\right)\left(b+n-n_{o}\right)\right]} d \lambda \\
\hat{p \pi_{s}} & =\frac{1}{\beta\left[\left(a+n_{o}\right)\left(b+n-n_{o}\right)\right]} \int_{0}^{1} \lambda^{\left(a+n_{o}+1\right)-1}(1-\lambda)^{\left(b+n-n_{o}\right)-1} d \lambda \\
& =\frac{1}{\beta\left[\left(a+n_{o}\right)\left(b+n-n_{o}\right)\right]} \beta\left[\left(a+n_{o}+1\right)\left(b+n-n_{o}\right)\right] \\
& =\frac{\Gamma(a+b+n)}{\Gamma\left(a+n_{o}\right) \Gamma\left(b+n-n_{o}\right)} \times \frac{\Gamma\left(a+n_{o}+1\right) \Gamma\left(b+n-n_{o}\right)}{\Gamma(a+b+n+1)}
\end{aligned}
$$

By definition, $\Gamma(\alpha)=(\alpha-1)$ !. So, the up equation yields

$$
\hat{\pi}_{s}=\frac{a+n_{o}}{p(a+b+n)} .
$$

The variance of Bayesian estimator of the proposed RRM follows by taking the variance of (65) as

$$
\begin{aligned}
\operatorname{Var}\left(\hat{\pi}_{s}\right) & =\operatorname{Var}\left[\frac{a+n_{o}}{p(a+b+n)}\right] \\
& =\frac{1}{[p(a+b+n)]^{2}} n p \pi_{s}\left[p\left(1-\pi_{s}\right)+(1-p)\right] .
\end{aligned}
$$

After some algebraic operations, equation (66) gives

$$
\hat{\operatorname{Var}}\left(\hat{\pi}_{s}\right)=\frac{n \pi_{s}\left(1-\pi_{s}\right)}{(a+b+n)^{2}}+\frac{n(1-p) \pi_{s}}{p(a+b+n)^{2}} .
$$

For large $n$, the influence of $a$ and $b$ in equations (65) and (67) become negligible because the weight $\frac{n}{a+b+n} \rightarrow 1$. Consequently, the posterior mean and variance are getting closer to classical (maximum likelihood) estimators in equations (20) and (23), respectively.

\section{Results and Discussion}

This section presents analysis of the simulated data of different sample sizes that are binomially distributed (with parameters $(n, p))$ to validate the developed model. [33] established that the best average value of $p$ across RR studies is 0.7 . However, this study considered values of $p$ within the range $[0.6,0.8]$ at equal step of 0.1 . The proposed model was estimated and variance comparison was done to examine its performance with some other existing models using [34](R-software version 3.6.0) and "LearnBayes" package in R-software authored by [35]. The tables and figures showing the results are presented below and the results discussion follows:

\subsection{Discussion}

In terms of minimum variance, column (2) and column (6) of Tables 4, 5 and 6 show that the proposed RR model is more efficient and precise than [7] original RRM. In a situation where $\pi_{u} \neq 0$ and $\pi_{s}$ in the neighbourhood of 0.6 and below, columns 4 and 6 of Tables 4, 5 and 6 reveal that the proposed MFRRM is more efficient than [12] original FRRM. In addition, Table 7 depicts that if $\pi_{u}=0,[12]$ and the proposed model produce same results not only for $\operatorname{Var}\left(\hat{\pi}_{s}\right)$ but also $\hat{\pi}_{s}$ across different sample sizes regardless of pre-assigned randomization parameter $(p)$. Mangat's procedure is more efficient but less effective when compare with the earlier similar estimators (models). The proposed model is more protective than [15] RRM. Moreover, the results demonstrate that it is more efficient given the following condition: when $\pi_{s}$ assume values $\leq \frac{1}{2}$. This condition is already established in equation (48). A more detailed insight of the results is given in columns 5 and 6 of Tables 4, 5 and 6 that showed conditional efficiency of the two designs. When $\pi_{s}>\frac{1}{2}$, Mangat is more efficient (see Table 6).

Like [11] model, especially if the sensitive variable under study is highly rampant, the proposed estimator has tendency to produce estimate whose value is greater than unity. Tables 1, 2 and 3 confirmed this possibility. Second column of Table 9 also show that Kim and Warde Warner's stratified RRM has the same property of producing proportion greater than unity.

Furthermore, Bayesian estimation of both Warner's and the proposed MFRRM provide relatively more precise estimators than their classical (maximum likelihood) estimators, conditional on the sample size $(n)$ and total of yes-responses $\left(n_{o}\right)$. But in large samples, Bayesian estimator asymptotically approaching the classical estimators (refer to columns 3 and 4, 7 and 8 of Table 1 through 3; columns 2 and 3, 6 and 7 of Table 4 through 6 for details). To update the user's prior knowledge about $\pi_{s}$, the study considers posterior of immediate sample size as a new prior in the subsequent sampling to generate a new posterior which produce Figure 3. Figure 3 above reveals that the posterior density compromises between the initial prior beliefs and the information in the data.

Again, using information from Table 8, the numerical illustration shows that the proposed stratified MFRRM is more efficient than Kim and Warde Warner's stratified RRM as $\operatorname{Var}\left(\hat{\pi}_{\text {stpropo }}\right)=$ $0.0007313622<\operatorname{Var}\left(\hat{\pi}_{\text {skw }}\right)=0.002960187$ (compare column 4 of Tables 10 and 14). In addition, the proposed model is less efficient when compared with mixed stratified RRM of [17] (see column 4 of Tables 12 and 14). Both Warner's stratified and mixed stratified RRMs have tendency to produce negative estimate of $\hat{\pi}_{s h}$ which has a seeming distance from reality. The possibility of negative stratum proportion is well known from their theoretical results particularly when $\hat{\lambda}_{h}+p_{h}<1$ (see first row, second column of Table 11). Theoretically and empirically, the proposed stratified RRM can not produce negative $\hat{\pi}_{s h}$ (refer to equation (27) and Table 13 for confirmation).

\section{Concluding Remarks}

This study proposed a modified version of FRRM for designing surveys that will elicit response to sensitive issues from sample units. Although extant models such as [7] original RRM, [12] original FRRM, and [15] FRRM among others have focused on improving response in RR surveys exists in the literature. However, designs of some of these models are not 
Table 1: Estimate of $\pi_{s}$ for the proposed model and some existing models when $p=0.6, \pi_{u}=0.75, a=10.2$ and $b=27.4$

\begin{tabular}{llcccccc}
\hline$n$ & $n_{o}$ & \multicolumn{2}{c}{ Warner RRM } & Boruch & Mangat & \multicolumn{2}{c}{ Proposed MFRRM } \\
\hline & & $\hat{\pi}_{\text {mle }}$ & $\hat{\pi}_{\text {bayesian }}$ & $\hat{\pi}_{\text {mle }}$ & $\hat{\pi}_{\text {mle }}$ & $\hat{\pi}_{\text {mle }}$ & $\hat{\pi}_{\text {bayesian }}$ \\
\hline 200 & 118 & 0.950000 & 0.6978114 & 0.4833333 & 0.3166667 & 0.9833333 & 0.8992705 \\
400 & 244 & 1.050000 & 0.9044790 & 0.5166667 & 0.3500000 & 1.0166667 & 0.9681597 \\
600 & 362 & 1.016667 & 0.9187578 & 0.5055556 & 0.3388889 & 1.0055556 & 0.9729193 \\
800 & 476 & 0.975000 & 0.9023400 & 0.4916667 & 0.3250000 & 0.9916667 & 0.9674467 \\
1000 & 591 & 0.955000 & 0.8970702 & 0.4850000 & 0.3183333 & 0.9850000 & 0.9656901 \\
10000 & 5949 & 0.974500 & 0.9684387 & 0.4915000 & 0.3248333 & 0.9915000 & 0.9894796 \\
100000 & 60303 & 1.015150 & 1.0145265 & 0.5050500 & 0.3383833 & 1.0050500 & 1.0048422 \\
1000000 & 600418 & 1.002090 & 1.0020281 & 0.5006967 & 0.3340300 & 1.0006967 & 1.0006760 \\
\hline
\end{tabular}

Table 2: Estimate of $\pi_{s}$ for the proposed model and some existing models when $p=0.7, \pi_{u}=0.75, a=10.2$ and $b=27.4$

\begin{tabular}{llcccccc}
\hline$n$ & $n_{o}$ & \multicolumn{2}{c}{ Warner RRM } & Boruch & \multicolumn{2}{c}{ Mangat } & \multicolumn{2}{c}{ Proposed MFRRM } \\
\hline & & $\hat{\pi}_{\text {mle }}$ & $\hat{\pi}_{\text {bayesian }}$ & $\hat{\pi}_{\text {mle }}$ & $\hat{\pi}_{\text {mle }}$ & $\hat{\pi}_{\text {mle }}$ & $\hat{\pi}_{\text {bayesian }}$ \\
\hline 200 & 135 & 0.9375000 & 0.7777778 & 0.6428571 & 0.5357143 & 0.9642857 & 0.8730159 \\
400 & 275 & 0.9687500 & 0.8793419 & 0.6607143 & 0.5535714 & 0.9821429 & 0.9310525 \\
600 & 416 & 0.9833333 & 0.9211104 & 0.6690476 & 0.5619048 & 0.9904762 & 0.9549202 \\
800 & 557 & 0.9906250 & 0.9429322 & 0.6732143 & 0.5660714 & 0.9946429 & 0.9673898 \\
1000 & 699 & 0.9975000 & 0.9587510 & 0.6771429 & 0.5700000 & 0.9985714 & 0.9764291 \\
10000 & 6958 & 0.9895000 & 0.9855244 & 0.6725714 & 0.5654286 & 0.9940000 & 0.9917282 \\
100000 & 70104 & 1.0026000 & 1.0021962 & 0.6800571 & 0.5729143 & 1.0014857 & 1.0012550 \\
1000000 & 700007 & 1.0000175 & 0.9999772 & 0.6785814 & 0.5714386 & 1.0000100 & 0.9999870 \\
\hline
\end{tabular}

Table 3: Estimate of $\pi_{s}$ for the proposed model and some existing models when $p=0.8, \pi_{u}=0.75, a=10.2$ and $b=27.4$

\begin{tabular}{llcccccc}
\hline$n$ & $n_{o}$ & \multicolumn{2}{c}{ Warner RRM } & Boruch & \multicolumn{2}{c}{ Mangat } & \multicolumn{2}{c}{ Proposed MFRRM } \\
\hline & & \multicolumn{1}{c}{$\hat{\pi}_{\text {mle }}$} & $\hat{\pi}_{\text {bayesian }}$ & $\hat{\pi}_{\text {mle }}$ & $\hat{\pi}_{\text {mle }}$ & $\hat{\pi}_{\text {mle }}$ & $\hat{\pi}_{\text {bayesian }}$ \\
\hline 200 & 151 & 0.9250000 & 0.7974186 & 0.7562500 & 0.6937500 & 0.9437500 & 0.8480640 \\
400 & 308 & 0.9500000 & 0.8785801 & 0.7750000 & 0.7125000 & 0.9625000 & 0.9089351 \\
600 & 470 & 0.9722222 & 0.9218946 & 0.7916667 & 0.7291667 & 0.9791667 & 0.9414210 \\
800 & 624 & 0.9666667 & 0.9286055 & 0.7875000 & 0.7250000 & 0.9750000 & 0.9464542 \\
1000 & 785 & 0.9750000 & 0.9439733 & 0.7937500 & 0.7312500 & 0.9812500 & 0.9579800 \\
10000 & 7977 & 0.9961667 & 0.9928801 & 0.8096250 & 0.7471250 & 0.9971250 & 0.9946601 \\
100000 & 80061 & 1.0010167 & 1.0006851 & 0.8132625 & 0.7507625 & 1.0007625 & 1.0005138 \\
1000000 & 800137 & 1.0002283 & 1.0001952 & 0.8126712 & 0.7501713 & 1.0001712 & 1.0001464 \\
\hline
\end{tabular}

Table 4: Performance comparison of the proposed model with some existing models when $p=0.7, \pi_{s}=0.1, \pi_{u}=0.75, a=10.2$ and $b=27.4$

\begin{tabular}{llccccc}
\hline$n$ & \multicolumn{2}{c}{ Warner RRM } & Boruch & Mangat & \multicolumn{2}{c}{ Proposed MFRRM } \\
\hline & $V\left(\hat{\pi}_{\text {mle }}\right)$ & $V\left(\hat{\pi}_{\text {bayesian }}\right)$ & $V\left(\hat{\pi}_{\text {mle }}\right)$ & $V\left(\hat{\pi}_{\text {mle }}\right)$ & $V\left(\hat{\pi}_{\text {mle }}\right)$ & $V\left(\hat{\pi}_{\text {bayesian }}\right)$ \\
\hline 200 & 0.0070125000 & $4.968668 \mathrm{e}-03$ & $2.122194 \mathrm{e}-03$ & $2.378571 \mathrm{e}-03$ & $6.642857 \mathrm{e}-04$ & $4.706760 \mathrm{e}-04$ \\
400 & 0.0035062500 & $2.929599 \mathrm{e}-03$ & $1.061097 \mathrm{e}-03$ & $1.189286 \mathrm{e}-03$ & $3.321429 \mathrm{e}-04$ & $2.775174 \mathrm{e}-04$ \\
600 & 0.0023375000 & $2.069939 \mathrm{e}-03$ & $7.073980 \mathrm{e}-04$ & $7.928571 \mathrm{e}-04$ & $2.214286 \mathrm{e}-04$ & $1.960828 \mathrm{e}-04$ \\
800 & 0.0017531250 & $1.599262 \mathrm{e}-03$ & $5.305485 \mathrm{e}-04$ & $5.946429 \mathrm{e}-04$ & $1.660714 \mathrm{e}-04$ & $1.514961 \mathrm{e}-04$ \\
1000 & 0.0014025000 & $1.302696 \mathrm{e}-03$ & $4.244388 \mathrm{e}-04$ & $4.757143 \mathrm{e}-04$ & $1.328571 \mathrm{e}-04$ & $1.234028 \mathrm{e}-04$ \\
10000 & 0.0001402500 & $1.392012 \mathrm{e}-04$ & $4.244388 \mathrm{e}-05$ & $4.757143 \mathrm{e}-05$ & $1.328571 \mathrm{e}-05$ & $1.318637 \mathrm{e}-05$ \\
100000 & 0.0000140250 & $1.401446 \mathrm{e}-05$ & $4.244388 \mathrm{e}-06$ & $4.757143 \mathrm{e}-06$ & $1.328571 \mathrm{e}-06$ & $1.327573 \mathrm{e}-06$ \\
1000000 & 0.0000014025 & $1.402395 \mathrm{e}-06$ & $4.244388 \mathrm{e}-07$ & $4.757143 \mathrm{e}-07$ & $1.328571 \mathrm{e}-07$ & $1.328472 \mathrm{e}-07$ \\
\hline
\end{tabular}

effective and their estimators are not efficient. Based on the simulated results and other evidence from variance comparison, the proposed MFRRM outperformed some of these models. In terms of efficiency, the proposed model is preferred to [7] RRM as it portents or exhibits minimal variance. In compar- ison with [12] FRRM, the proposed MFFRRM is more efficient and also more flexible as it does not requires prior knowledge of $\pi_{u}$ unlike Boruch's original FRRM. To some extent, the proposed model is more efficient than [15] RRM. Its practicability trascends Mangat's as it is wholly stochastic in procedure thus 
Table 5: Performance comparison of the proposed model with some existing models when $p=0.7, \pi_{s}=0.4, \pi_{u}=0.75, a=10.2$ and $b=27.4$

\begin{tabular}{llccccc}
\hline$n$ & \multicolumn{2}{c}{ Warner RRM } & Boruch & Mangat & \multicolumn{2}{c}{ Proposed MFRRM } \\
\hline & $V\left(\hat{\pi}_{\text {mle }}\right)$ & $V\left(\hat{\pi}_{\text {bayesian }}\right)$ & $V\left(\hat{\pi}_{\text {mle }}\right)$ & $V\left(\hat{\pi}_{\text {mle }}\right)$ & $V\left(\hat{\pi}_{\text {mle }}\right)$ & $V\left(\hat{\pi}_{\text {bayesian }}\right)$ \\
\hline 200 & 0.0077625000 & $5.500077 \mathrm{e}-03$ & $2.550765 \mathrm{e}-03$ & $2.485714 \mathrm{e}-03$ & $2.057143 \mathrm{e}-03$ & $1.457577 \mathrm{e}-03$ \\
400 & 0.0038812500 & $3.242926 \mathrm{e}-03$ & $1.275383 \mathrm{e}-03$ & $1.242857 \mathrm{e}-03$ & $1.028571 \mathrm{e}-03$ & $8.594088 \mathrm{e}-04$ \\
600 & 0.0025875000 & $2.291323 \mathrm{e}-03$ & $8.502551 \mathrm{e}-04$ & $8.285714 \mathrm{e}-04$ & $6.857143 \mathrm{e}-04$ & $6.072242 \mathrm{e}-04$ \\
800 & 0.0019406250 & $1.770306 \mathrm{e}-03$ & $6.376913 \mathrm{e}-04$ & $6.214286 \mathrm{e}-04$ & $5.142857 \mathrm{e}-04$ & $4.691493 \mathrm{e}-04$ \\
1000 & 0.0015525000 & $1.442021 \mathrm{e}-03$ & $5.101531 \mathrm{e}-04$ & $4.971429 \mathrm{e}-04$ & $4.114286 \mathrm{e}-04$ & $3.821506 \mathrm{e}-04$ \\
10000 & 0.0001552500 & $1.540891 \mathrm{e}-04$ & $5.101531 \mathrm{e}-05$ & $4.971429 \mathrm{e}-05$ & $4.114286 \mathrm{e}-05$ & $4.083520 \mathrm{e}-05$ \\
100000 & 0.0000155250 & $1.551333 \mathrm{e}-05$ & $5.101531 \mathrm{e}-06$ & $4.971429 \mathrm{e}-06$ & $4.114286 \mathrm{e}-06$ & $4.111194 \mathrm{e}-06$ \\
1000000 & 0.0000015525 & $1.552383 \mathrm{e}-06$ & $5.101531 \mathrm{e}-07$ & $4.971429 \mathrm{e}-07$ & $4.114286 \mathrm{e}-07$ & $4.113976 \mathrm{e}-07$ \\
\hline
\end{tabular}

Table 6: Performance comparison of the proposed model with some existing models when $p=0.7, \pi_{s}=0.6, \pi_{u}=0.75, a=10.2$ and $b=27.4$

\begin{tabular}{llccccc}
\hline$n$ & \multicolumn{2}{c}{ Warner RRM } & Boruch & Mangat & \multicolumn{2}{c}{ Proposed MFRRM } \\
\hline & $V\left(\hat{\pi}_{\text {mle }}\right)$ & $V\left(\hat{\pi}_{\text {bayesian }}\right)$ & $V\left(\hat{\pi}_{\text {mle }}\right)$ & $V\left(\hat{\pi}_{\text {mle }}\right)$ & $V\left(\hat{\pi}_{\text {mle }}\right)$ & $V\left(\hat{\pi}_{\text {bayesian }}\right)$ \\
\hline 200 & 0.0077625000 & $5.500077 \mathrm{e}-03$ & $2.501020 \mathrm{e}-03$ & $2.057143 \mathrm{e}-03$ & $2.485714 \mathrm{e}-03$ & $1.761239 \mathrm{e}-03$ \\
400 & 0.0038812500 & $3.242926 \mathrm{e}-03$ & $1.250510 \mathrm{e}-03$ & $1.028571 \mathrm{e}-03$ & $1.242857 \mathrm{e}-03$ & $1.038452 \mathrm{e}-03$ \\
600 & 0.0025875000 & $2.291323 \mathrm{e}-03$ & $8.336735 \mathrm{e}-04$ & $6.857143 \mathrm{e}-04$ & $8.285714 \mathrm{e}-04$ & $7.337293 \mathrm{e}-04$ \\
800 & 0.0019406250 & $1.770306 \mathrm{e}-03$ & $6.252551 \mathrm{e}-04$ & $5.142857 \mathrm{e}-04$ & $6.214286 \mathrm{e}-04$ & $5.668888 \mathrm{e}-04$ \\
1000 & 0.0015525000 & $1.442021 \mathrm{e}-03$ & $5.002041 \mathrm{e}-04$ & $4.114286 \mathrm{e}-04$ & $4.971429 \mathrm{e}-04$ & $4.617653 \mathrm{e}-04$ \\
10000 & 0.0001552500 & $1.540891 \mathrm{e}-04$ & $5.002041 \mathrm{e}-05$ & $4.114286 \mathrm{e}-05$ & $4.971429 \mathrm{e}-05$ & $4.934253 \mathrm{e}-05$ \\
100000 & 0.0000155250 & $1.551333 \mathrm{e}-05$ & $5.002041 \mathrm{e}-06$ & $4.114286 \mathrm{e}-06$ & $4.971429 \mathrm{e}-06$ & $4.967692 \mathrm{e}-06$ \\
1000000 & 0.0000015525 & $1.552383 \mathrm{e}-06$ & $5.002041 \mathrm{e}-07$ & $4.114286 \mathrm{e}-07$ & $4.971429 \mathrm{e}-07$ & $4.971055 \mathrm{e}-07$ \\
\hline
\end{tabular}

Table 7: Comparison of original FRRM and the Proposed MFRRM when $p=0.7$ and $\pi_{u}=0$

\begin{tabular}{llcccc}
\hline & \multicolumn{3}{c}{ Boruch original FRRD } & \multicolumn{2}{c}{ Proposed MFRRD } \\
\hline$n$ & $n_{o}$ & $\hat{\pi}_{\text {mle }}$ & $V\left(\hat{\pi}_{\text {mle }}\right)$ & $\hat{\pi}_{\text {mle }}$ & $V\left(\hat{\pi}_{\text {mle }}\right)$ \\
\hline 200 & 135 & 0.9642857 & $2.514286 \mathrm{e}-03$ & 0.9642857 & $2.514286 \mathrm{e}-03$ \\
400 & 275 & 0.9821429 & $1.257143 \mathrm{e}-03$ & 0.9821429 & $1.257143 \mathrm{e}-03$ \\
600 & 416 & 0.9904762 & $8.380952 \mathrm{e}-04$ & 0.9904762 & $8.380952 \mathrm{e}-04$ \\
800 & 557 & 0.9946429 & $6.285714 \mathrm{e}-04$ & 0.9946429 & $6.285714 \mathrm{e}-04$ \\
1000 & 699 & 0.9985714 & $5.028571 \mathrm{e}-04$ & 0.9985714 & $5.028571 \mathrm{e}-04$ \\
10000 & 6958 & 0.9940000 & $5.028571 \mathrm{e}-05$ & 0.9940000 & $5.028571 \mathrm{e}-05$ \\
100000 & 70104 & 1.0014857 & $5.028571 \mathrm{e}-06$ & 1.0014857 & $5.028571 \mathrm{e}-06$ \\
1000000 & 700007 & 1.0000100 & $5.028571 \mathrm{e}-07$ & 1.0000100 & $5.028571 \mathrm{e}-07$ \\
\hline
\end{tabular}

Table 8: Samples and strata sizes

\begin{tabular}{lllllll}
\hline Strata & $N_{h}$ & $n_{h}$ & $p_{h}$ & $n_{o h}$ & $W_{h}$ & $\hat{\lambda}_{h}$ \\
\hline 1 & 876 & 69 & 0.4 & 27 & 0.08981852 & 0.3913043 \\
2 & 2412 & 118 & 0.6 & 51 & 0.24730852 & 0.4322034 \\
3 & 3012 & 279 & 0.7 & 115 & 0.30882805 & 0.4121864 \\
4 & 3453 & 288 & 0.8 & 102 & 0.35404491 & 0.3541667 \\
\hline Total & $\mathbf{9 7 5 3}$ & $\mathbf{7 5 4}$ & & $\mathbf{1 . 0 0}$ & \\
\hline
\end{tabular}

Table 9: Computational procedure of Kim and Warde Warner's stratified RRM

\begin{tabular}{lcccc}
\hline Strata & $\hat{\pi}_{s h}$ & $W_{h} \hat{\pi}_{s h}$ & $\hat{\phi}_{s h}$ & $W_{h} \phi_{s h}$ \\
\hline 1 & 1.0434783 & 0.09372367 & 2.440211 & 0.2191762 \\
2 & 0.1610169 & 0.03982086 & 2.476911 & 0.6125613 \\
3 & 0.2804659 & 0.08661575 & 1.230571 & 0.3800348 \\
4 & 0.2569444 & 0.09096987 & 0.797100 & 0.2822092 \\
\hline Total & & $\mathbf{0 . 3 1 1 1 3 0 2}$ & & $\mathbf{1 . 4 9 3 9 8 2}$ \\
\hline
\end{tabular}



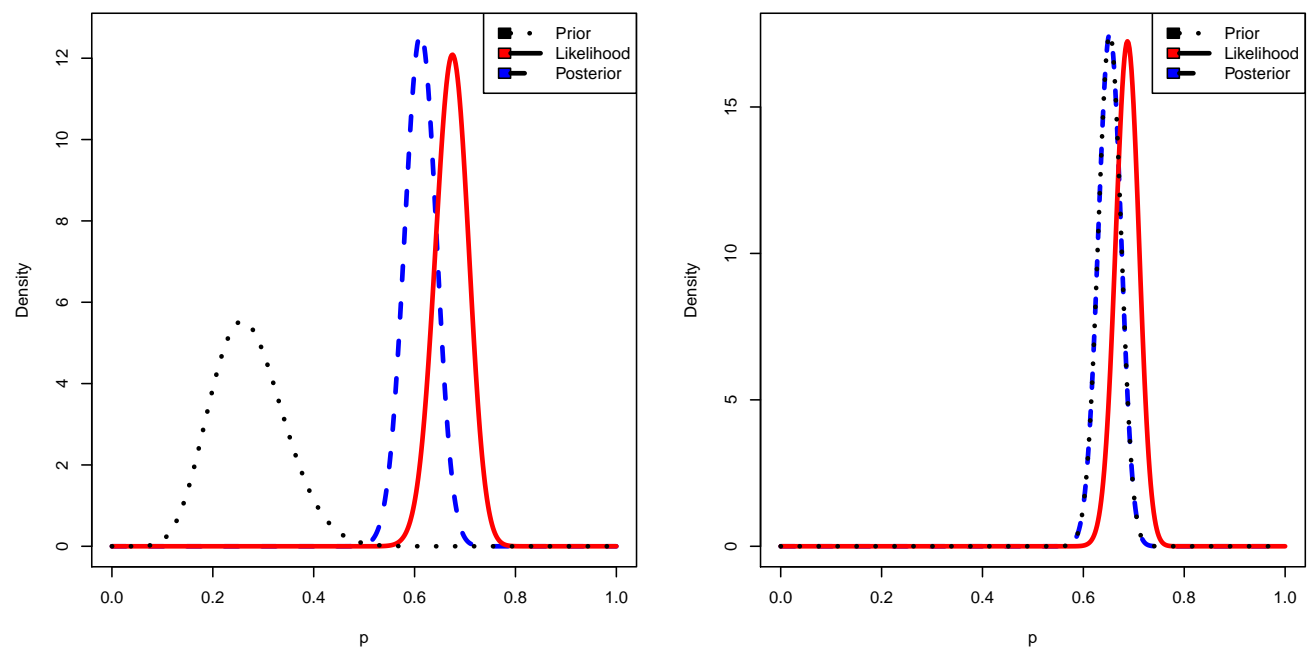

Figure 3: The Prior, the Likelihood and the Posterior for $n=200$ and $n=400$

Table 10: Summary of Kim and Warde Warner's stratified RRM

\begin{tabular}{lcccc}
\hline$N$ & $n$ & $\hat{\pi}_{s}$ & $\operatorname{Var}\left(\hat{\pi}_{s}\right)$ & $95 \%$ CI \\
\hline 9753 & 754 & 0.3111302 & 0.002960187 & $0.2044932,0.4177671$ \\
\hline
\end{tabular}

Table 11: Computational procedure of mixed stratified RRM

\begin{tabular}{lcccc}
\hline Strata & $\hat{\pi}_{s h}$ & $W_{h} \hat{\pi}_{s h}$ & $\hat{\phi}_{s h}$ & $W_{h} \phi_{s h}$ \\
\hline 1 & -0.40831758 & -0.03667448 & 1.2399337 & 0.1113690 \\
2 & 0.04639471 & 0.01147381 & 0.8246085 & 0.2039327 \\
3 & 0.13211914 & 0.04080210 & 0.6975762 & 0.2154311 \\
4 & 0.13650174 & 0.04832774 & 0.5777054 & 0.2045337 \\
\hline Total & & $\mathbf{0 . 0 6 3 9 2 9 1 7}$ & & $\mathbf{0 . 7 3 5 2 6 6 5}$ \\
\hline
\end{tabular}

Table 12: Summary of mixed stratified RRM

\begin{tabular}{lcccc}
\hline$N$ & $n$ & $\hat{\pi}_{s}$ & $\operatorname{Var}\left(\hat{\pi}_{s}\right)$ & $95 \%$ CI \\
\hline 9753 & 754 & 0.06392917 & 0.0007169984 & $0.01144755,0.1164108$ \\
\hline
\end{tabular}

Table 13: Computational procedure of the proposed stratified MFRRM

\begin{tabular}{lcccc}
\hline Strata & $\hat{\pi}_{s h}$ & $W_{h} \hat{\pi}_{s h}$ & $\hat{\phi}_{s h}$ & $W_{h} \phi_{s h}$ \\
\hline 1 & 0.9782609 & 0.08786594 & 1.2201057 & 0.1095881 \\
2 & 0.7203390 & 0.17814597 & 0.8256372 & 0.2041871 \\
3 & 0.5888377 & 0.18184960 & 0.7031834 & 0.2171628 \\
4 & 0.4427083 & 0.15673863 & 0.5978250 & 0.2116569 \\
\hline Total & & $\mathbf{0 . 6 0 4 6 0 0 1}$ & & $\mathbf{0 . 7 4 2 5 9 4 8}$ \\
\hline
\end{tabular}

Table 14: Summary of proposed stratified MFRRM

\begin{tabular}{lcccc}
\hline$N$ & $n$ & $\hat{\pi}_{\text {stpropo }}$ & $\operatorname{Var}\left(\hat{\pi}_{\text {stpropo }}\right)$ & $95 \%$ CI \\
\hline 9753 & 754 & 0.6046001 & 0.0007313622 & $0.5515954,0.6576048$ \\
\hline
\end{tabular}

avoiding any posiible identification of individual status. The motivation for adopting existing RRT in sensitive related survey is protection of respondents' privacy, and for the fact that the proposed MFRRM is more efficient, flexible and practical over some of the leading RRT, the study suggest its adoption.

The proposed model argues that majority of people have a wrong intuition of the concepts of probabilities. This ignorance was 
used to the advantage of the survey under consideration by making the subjective privacy protection larger than the true statistics privacy protection. Though, the proposed design is still in accordance with the principle of RRT, since the identity of the respondent is not required and the randomization process makes it impossible to know or even guess the response of a particular respondent. It is imperative to recall that RRT was introduced to protect respondents' privacy and encourage them to divulge truthful answers rather than trapping them with probability or mathematical techniques to trace their real status on the sensitive issue(s) understudy as suggested by [37-38], and the proposed model. The study however suggests further quest for more robust RRM in view of the possibility that respondents can subsequently assimilate the randomization process thereby increasing false or evasive response, or at worst refusal to participate in such survey.

\section{Acknowledgements}

The authors are grateful to the referee and editor for their valuable comments and suggestions.

\section{References}

[1] G. N. Amahia, "Factors, Prevention and Correction Methods for NonResponse in Sample Surveys", Central Bank of Nigeria Journal of Applied Statistics 1 (2010) 79.

[2] K. Hong, J. Yum \& H. Lee, "A Stratified Randomized Response Technique", The Korean Journal of Applied Statistics, 7 (1994) 141.

[3] G. S. Lee, K. H. Hong, J. M. Kim \& C. K. Son, "Estimation of the Proportion of a Sensitive Attribute Based on a Two-Stage Randomized Response Model with Stratified Unequal Probability Sampling”, Brazilian Journal of Probability and Statistics, 28 (2014) 381.

[4] S. Sudman \& N. M. Bradburn, "Asking Questions: A Practical Guide to Questionnaire Design", San Fransisco, CA: Jossey-Bass (1982).

[5] K. A. Rasinski, G. B. Willis, Baldwin, W. C. Yeh \& L. Lee, "Methods of Data Collection, Perceptions of Risks and Losses, and Motivation to give Truthful Answers to Sensitive Survey Questions", Applied Cognitive Psychology, 13 (1999) 465.

[6] C. N. Bouza, C. Herrera \& P. G. Mitra, "A Review of Randomized Responses Procedures: The Qualitative Variable Case", Revista Investigación 31 (2010) 240.

[7] S. L. Warner, "Randomized Response: A Survey Technique for Eliminating Evasive Answer Bias", Journal of American Statistical Association, 60(1965) 63, DOI: 10.2307/2283137, http://www.jstor.org/stable/2283137.

[8] G. J. L. M. Lensvelt-Mulders, J. J. Hox \& P. G. M. Van-derHeijden, "How to Improve the Efficiency of Randomized Response Designs", Quality and Quantity: Springer (2005) 39 (2005) 253, DOI: $10.1007 / \mathrm{s} 11135-004-0432-3$.

[9] A. C. Y. Chong, A. M. Y. Chu, M. K. P. So \& R. S. W. Chung, "Asking Sensitive Questions Using the Randomized Response Approach in Public Health Research: An Empirical Study on the Factors of Illegal Waste Disposal", International Journal of Environmental and Public Health 16 (2019) 1, doi:10.3390/ijerph16060970.

[10] D. G. Horvitz, B. V. Shah \& W. S. Simmons, "The Unrelated Question Randomized Response Model, Proceedings in the Social Science Section", American Statistical Association, (1967) 65.

[11] B. G. Greenberg, A. L. Abul-Ela, W. R. Simmons \& D. G. Horvitz, "The unrelated question Randomized Response Model: Theoretical Framework", Journal of the American Statistical Association, 64 (1969) 520, https://doi.org/10.1080/01621459.1969.10500991.

[12] R. F. Boruch, "Assuring Confidentiality of Responses in Social Research: A Note on Strategies", The American Sociologist, 6 (1971) 308.
[13] J. J. A. Moors, "Optimization of the Unrelated Question Randomized Response Model", Journal of the American Statistical Association, 66 (1971) 627, DOI: 10.1080/01621459.1971.10482320.

[14] N. S. Mangat \& R. Singh, An Alternative Randomized Response Procedure, Biometrika, 77 (1990) 439, https://doi.org/10.1093/biomet/77.2.439.

[15] N. S. Mangat, "An Improved Randomized Response Strategy", Journal of Royal Statistical Society: Series B, 56 (1994) 93.

[16] J. M. Kim \& W. D. Warde, "A stratified Warner's Randomized Response Model", Elsevier Journal of Statistical Planning and Inference, 120 (2004) 155.

[17] A. Usman \& I. O. Oshungade, ”A Mixed-stratified Randomized Response Model for HIV Seroprevalence Surveys", Research Journal of Mathematics and Statistics, 4 (2012) 70.

[18] D. W. Gingerich, "Understanding Off-the-books Politics: Conducting Inference on the Determinants of Sensitive Behavior with Randomized Response Surveys", Political Analysis, 18 (2010) 349.

[19] J. A. Fox \& P. E. Tracy (1986), "Randomized Response: A Method for Sensitive Surveys", Beverly Hills, CA: Sage.

[20] G. Blair, K. Imai \& Y-Y Zhou, "Design and Analysis of the Randomized Response Technique", Journal of the American Statistical Association, 110 (2015) 1304, DOI: 10.1080/01621459.2015.1050028.

[21] B. Rosenfeld, K. Imai \& J. Shapiro, "An Empirical Validation Study of Popular Survey Methodologies for Sensitive Questions", American Journal of Political Science, $\mathbf{6 0}$ (2015) 783, doi: 10.1111/ajps.12205.

[22] J. H. Stubbe, A. M. Chorus, L. E. Frank, O. Hon \& P. G. Heijden, "Prevalence of Use of Performance Enhancing Drugs by Fitness Centre Members", Drug Testing and Analysis, 6 (2014) 434.

[23] I. Krumpal, "Estimating the Prevalence of Xenophobia and Anti-semitism in Germany: A Comparison of Randomized Response and Direct Questioning", Social Science Research, 41 (2012) 1387.

[24] F. A. St-John, A. M. Keane, G. Edwards-Jones, L. Jones, R. W. Yarnell \& J. P. Jones, ”Identifying Indicators of Illegal Behaviour: Carnivore Killing in Human-Managed Landscapes", Proceedings of the Royal Society B: Biological Sciences, 279 (2012) 804.

[25] H. Elffers, P. Van Der Heijden \& M. Hezemans, "Explaining Regulatory Non-compliance: A Survey Study of Rule Transgression For Two Dutch Instrumental Laws, Applying the Randomized Response Method", Journal of Quantitative Criminology, 19 (2003) 409, DOI: 10.1023/B:JOQC.0000005442.96987.9e.

[26] G. Diana, S. Riaz \& J. Shabbir, "Hansen and Hurwitz Estimator with Scrambled Response on the Second Call", Journal of Applied Statistics, 41 (2013)3, 596-611, http://dx.doi.org/10. 1080/02664763.2013.846305.

[27] O. S. Ewemooje \& G. N. Amahia, "Improved Randomized Response Technique for Two Sensitive Attributes", Afrika Statistika, 10 (2015) 839, DOI: $10.16929 / \mathrm{as} / 2015.639 .78$.

[28] W. G. Cochran, "Sampling Techniques", 3rd edition, New York: John Wiley and Sons (1977), MR 0474575.

[29] R. L. Winkler \& L. A. Franklin, "Warner's Randomized Response Model: A Bayesian Approach", Journal of the American Statistical Association, 74 (1979) 207, DOI: 10.2307/2286752, https://www.jstor.org/stable/2286752.

[30] Z. Hussain, J. Shabbir \& M. Riaz, "Bayesian Estimation using Warner's Randomized Response Model through Simple and Mixture Prior Distributions", Communications in Statistics-Simulation and Computation, 40 (2010) 147, DOI: 10.1080/03610918.2010.532897.

[31] D. Fink, "A Compendium of Conjugate Priors: Environmental Statistics Group", Department of Biology, Montana state university Bozeman (1997), MT 59717.

[32] F. J. Anscombe, "Bayesian Statistics", Journal of Royal Statistical Society, 25 (1962) 1.

[33] A. Quantember, "A Standardization of Randomized Response Strategies", Survey Methodology, 35 (2009) 143.

[34] R Core Team, "R: A language and environment for statistical computing", R Foundation for Statistical Computing, Vienna, Austria (2019), URL= https://www.R-project.org/.

[35] Jim Albert, "LearnBayes: Functions for Learning Bayesian Inference", R package version 3.6.0 (2018), https://CRAN.Rproject.org/package $=$ LearnBayes.

[36] J. M. Kim, J. M. Tebbs \& An Seung-Won, "Extensions of Mangat's Randomized-Response Model", Elsevier Journal of Statistical Planning 
and Inference, 136 (2006) 1554.

[37] P. G. M. Van der Heijden, G. Van Gils, J. Bouts, \& J. J. Hox, "A Comparison of Randomized Response, Computer-assisted self-interview, and Face-to-Face Direct Questioning", Sociological Methods and Research,
28 (2000) 505.

[38] H. Zawar \& K. Khushnoor, "On Estimation of Sensitive Mean Using Scrambled Data", World Applied Sciences Journal, 23 (2013) 1201. 